\title{
Spin-phonon coupling and dynamic zero-field splitting contributions to spin conversion processes in iron(II) complexes
}

Cite as: J. Chem. Phys. 152, 204306 (2020); https://doi.org/10.1063/5.0006361

Submitted: 02 March 2020 . Accepted: 07 May 2020 . Published Online: 27 May 2020

Nicholas J. Higdon (D), Alexandra T. Barth (D), Patryk T. Kozlowski, and Ryan G. Hadt (D)

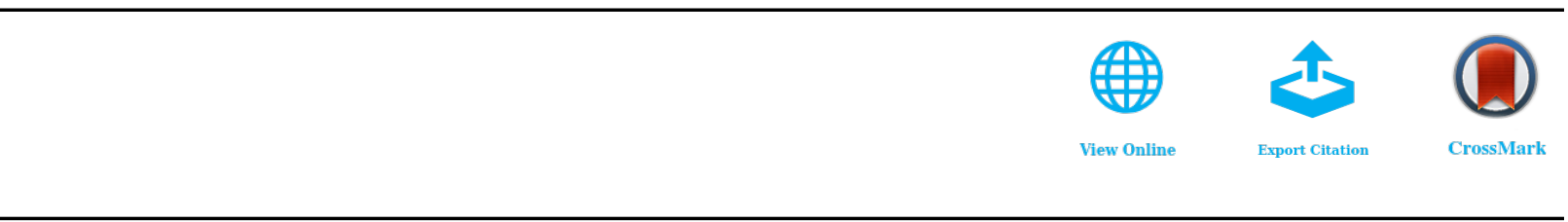

\section{Lock-in Amplifiers up to $600 \mathrm{MHz}$}

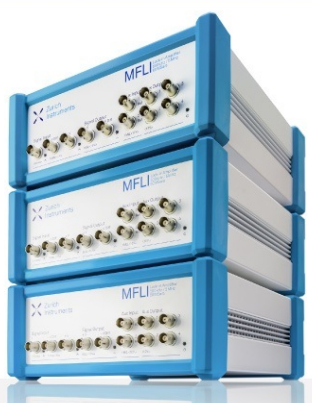




\title{
Spin-phonon coupling and dynamic zero-field splitting contributions to spin conversion processes in iron(II) complexes
}

\author{
Cite as: J. Chem. Phys. 152, 204306 (2020); doi: 10.1063/5.0006361 \\ Submitted: 2 March 2020 - Accepted: 7 May 2020 • \\ Published Online: 27 May 2020
}

Nicholas J. Higdon, (D) Alexandra T. Barth, (D) Patryk T. Kozlowski, and Ryan G. Hadt ${ }^{\text {a) }}$ (D)

\begin{abstract}
AFFILIATIONS
Division of Chemistry and Chemical Engineering, Arthur Amos Noyes Laboratory of Chemical Physics, California Institute of Technology, Pasadena, California 91125, USA
\end{abstract}

Note: This paper is part of the JCP Emerging Investigators Special Collection.

author to whom correspondence should be addressed: rghadt@caltech.edu

\begin{abstract}
Magnetization dynamics of transition metal complexes manifest in properties and phenomena of fundamental and applied interest [e.g., slow magnetic relaxation in single molecule magnets, quantum coherence in quantum bits (qubits), and intersystem crossing (ISC) rates in photophysics]. While spin-phonon coupling is recognized as an important determinant of these dynamics, additional fundamental studies are required to unravel the nature of the coupling and, thus, leverage it in molecular engineering approaches. To this end, we describe here a combined ligand field theory and multireference $a b$ initio model to define spin-phonon coupling terms in $\mathrm{S}=2$ transition metal complexes and demonstrate how couplings originate from both the static and dynamic properties of ground and excited states. By extending concepts to spin conversion processes, ligand field dynamics manifest in the evolution of the excited state origins of zero-field splitting (ZFS) along specific normal mode potential energy surfaces. Dynamic ZFSs provide a powerful means to independently evaluate contributions from spin-allowed and/or spin-forbidden excited states to spin-phonon coupling terms. Furthermore, ratios between various intramolecular coupling terms for a given mode drive spin conversion processes in transition metal complexes and can be used to analyze the mechanisms of ISC. Variations in geometric structure strongly influence the relative intramolecular linear spin-phonon coupling terms and will define the overall spin state dynamics. While the findings of this study are of general importance for understanding magnetization dynamics, they also link the phenomenon of spin-phonon coupling across fields of single molecule magnetism, quantum materials/qubits, and transition metal photophysics.
\end{abstract}

Published under license by AIP Publishing. https://doi.org/10.1063/5.0006361

\section{INTRODUCTION}

The coupling between structural and electronic degrees of freedom (e.g., spin-phonon coupling) plays critical roles in the dynamic properties of transition metal complexes. Relaxation phenomena in single molecule magnets (SMMs) and quantum bits (qubits) are thought to be strongly dependent on the coupling between vibrational modes and the electron spin $M_{S}$ sublevels derived from manyelectron molecular states (e.g., $M_{S}= \pm \frac{1}{2}$ for $S=\frac{1}{2}$ qubits and the zero-field split $M_{S}$ sublevels for most SMMs), which can effectively modulate their relative energies and mixings. ${ }^{1-8}$ In SMMs, these modulations can perturb magnetic anisotropy and result in lower effective barriers for relaxation $\left(\mathrm{U}_{\text {eff }}\right)$ and affect the tunneling of magnetization. ${ }^{4,5}$ In qubits, ligand field modulations can result in a finite variance in the splitting of the $M_{S}= \pm \frac{1}{2}$ sublevels (a reflector of molecular $g$ values), which can give rise to decoherence in a two-level system. ${ }^{8,9}$ Taken together, experimental and theoretical studies suggest that an increased fundamental understanding of spin-phonon coupling contributions to magnetic relaxation phenomena will provide a path toward the molecular engineering and eventual room temperature control of quantum materials for applications in quantum and classical computing. Additionally, increasing the temperature at which magnetic relaxation phenomena can be studied will open the door to more detailed studies of spin-phonon 
coupling in transition metal complexes. That is, at lower temperatures $(<\sim 80 \mathrm{~K})$, other relaxation mechanisms (e.g., direct, Raman, and Orbach) may dominate over local mode contributions, making it difficult to directly study spin-phonon couplings. ${ }^{10-1}$

While the phenomenon and consequences of spin-phonon coupling are of great importance for the quantum control of molecules and materials, this study is largely focused on the initial steps toward translating concepts and models to the study of spin conversion processes in transition metal complexes, including those exhibiting thermal spin crossover (SCO), light induced excited spin state trapping (LIESST), and/or ultrafast photo-triggered spin state switching in photomagnetic materials and photosensitizers. In these complexes, spin conversion processes can occur on an ultrafast timescale, even with large geometric changes. Thus, they are necessarily coupled to specific vibrational and/or phonon modes. These electron-phonon couplings are reflected in the potential energy surfaces (PESs) that connect the different spin states. Indeed, for photo-active transition metal complexes, ultrafast spectroscopic studies on six-coordinate $\mathrm{Fe}(\mathrm{II})$ complexes have demonstrated vibrational coherences that accompany photo-triggered spin state dynamics. ${ }^{14-21}$ This interplay between electronic and vibrational dynamics is an important factor for directing energy relaxation pathways within transition metal complexes. For example, spin-phonon coupling represents a significant hurdle for the development of earth-abundant photosensitizers made from Fe(II) for photochemical energy conversion pathways or photoredox catalysis. $^{22-24}$ In the first row transition metal complexes, excited ligand field states provide a direct relaxation pathway to the ground state. Conversely, $\mathrm{Ru}$ (II) and Ir(III) complexes lack low-energy ligand field transitions (i.e., the primogenic effect ${ }^{25}$ ), which gives rise to longer metal-to-ligand charge transfer (MLCT) excited state lifetimes.

Given these considerations, spin-phonon coupling represents a general, cross-cutting phenomenon of importance for the fundamental understanding of magnetization relaxation mechanisms in transition metal complexes for applications in spintronics, quantum materials, and/or photochemical energy conversion applications. However, it is important to develop combined experimental and computational methodologies to identify and quantify spinphonon active modes. ${ }^{3-7}$ Doing so will significantly aid our understanding of the origin(s) and nature of these couplings and, thus, allow for a more detailed rational synthetic approach to control dynamic magnetic properties in general.

While studies of spin-phonon couplings have largely considered the direct coupling between phonons and the $M_{S}$ sublevels, ${ }^{5,6}$ less focus has been on understanding the couplings through the lens of the transition metal excited state dynamics responsible for the experimental observables themselves [e.g., g values and zero-field splittings (ZFSs)]. ${ }^{26-28}$ For example, recent studies highlighted the role of ligand field excited state energies in affecting the relaxation rates of qubits at higher temperatures. ${ }^{3,8,29}$ The excited state energy contributions are exemplified through comparisons between four-coordinate $\mathrm{V}(\mathrm{IV}) \mathrm{O}$ (vanadyl), four-coordinate $\mathrm{Cu}(\mathrm{II})$, and six-coordinate $\mathrm{V}(\mathrm{IV})$ complexes as molecular qubit candidates. ${ }^{8}$ In six-coordinate V(IV) complexes, the presence of low energy ligand field transitions is thought to significantly increase spin-phonon coupling terms through the introduction of ground state orbital angular momentum through excited state spin orbit coupling (SOC). ${ }^{3,8}$ More explicitly, linear spin-phonon coupling terms in $S=\frac{1}{2}$ qubit candidates are predicted to exhibit a pseudoinverse square dependence on specific ligand field transition energies and a linear dependence on the associated linear excited state coupling terms. ${ }^{8}$ The relationships between linear spin-phonon and excited state coupling terms can also be used to predict and rationalize geometric dependencies on spin-lattice relaxation times in $\mathrm{Cu}(\mathrm{II})$ complexes and qubit candidates. ${ }^{8,10}$ Recognizing and understanding the important roles of excited state contributions to the ground state properties, thus, provide additional means to tune magnetic relaxation phenomena.

In contrast to considerations for qubits and SMMs, the switching between spin states in transition metal complexes introduces further interesting considerations: (1) geometric distortions upon spin conversion are typically more dramatic and sample more expansive regions of ground and excited PESs and (2) as the origins of ZFS derive from excited states and their geometric perturbations, the magnitudes and partial derivatives of ZFS can vary quite strongly along specific vibrational modes, which necessarily requires the invocation of spin-phonon coupling. More specifically, while the ZFS at an equilibrium geometry may be largely due to spinorbit interactions with one or two specific excited states [e.g., ${ }^{5} E_{g}$ in the case of $\mathrm{S}=2 \mathrm{Fe}(\mathrm{II})$, vide infra], the relative couplings among excited and ground states can change dramatically at different locations along PESs. This is especially important for considering spin conversion processes in transition metal complexes. Thus, both the rational design of molecular magnetic materials and the understanding of photochemical energy conversion pathways require a detailed knowledge of not only the coupling of vibrational degrees of freedom with ground state properties but also, of equal importance, the coupling of these vibrational degrees of freedom over the manifolds of ligand field excited states.

Here, we provide a detailed, combined ligand field theory and multireference $a b$ initio approach to understand the fundamental nature of spin-phonon couplings in $S>\frac{1}{2}$ transition metal complexes, with an emphasis on linking concepts in spin-phonon coupling between molecular magnetic materials and photoactive transition metal complexes. Similar to Mirzoyan and Hadt, ${ }^{8}$ this study shows that spin-phonon couplings derive from excited state coupling terms and are very sensitive to the absolute ligand field excited state energies. For specific vibrational modes, analyses can directly quantify spin-phonon coupling terms across Fe(II) complexes and demonstrate that couplings over the vibrational manifold are sensitive to the initial geometric structure of the transition metal complex (which governs the corresponding linear excited state coupling terms); accordingly, couplings can be tuned through structural perturbations to the first coordination sphere. Furthermore, while these complexes are in the ionic regime with limited ligand-metal covalency, the model described here predicts a reduction in spinphonon coupling terms with increased covalency, similar to discussions relating to qubits. ${ }^{8,30}$ In the limit of very low energy excited states or when considering orbitally degenerate excited states with in-state orbital angular momentum, significant deviations between the ligand field and $a b$ initio models described here can occur. Finally, we highlight the importance of the evolution of the splittings and mixings of the $M_{S}$ sublevels at intersystem crossings (ISCs) involved in spin conversion processes. While the ZFSs at $\mathrm{S}=2 \mathrm{Fe}(\mathrm{II})$ equilibrium geometries are largely due to a set of 
conserved ${ }^{5} E_{g}$ excited states, the nature of the couplings and the specific excited state contributions to the energetics and dynamical properties of the $M_{S}$ sublevels change dramatically along vibrational modes that facilitate ISC. The model and findings discussed here have broad impacts for the design of molecules and materials that exhibit important quantum phenomena at room temperature, as well as those involved in LIESST, SCO, photomagnetism, and solar energy conversion.

\section{RESULTS}

In the following Subsections II A-II D 2, a dynamic ligand field theory model of spin-phonon coupling terms is established that relates ground state axial ZFSs to the energetics of specific ligand field excited states. The model is then experimentally calibrated and evaluated using Fe(II) model complexes with welldefined first coordination spheres and magnetic properties. Finally, spin-phonon coupling terms and dynamics are extended to spin conversion processes.

\section{A. Dynamic ligand field theory of $S=2 \mathrm{Fe}(\mathrm{II})$ zero-field splitting}

As shown in Fig. 1, in $O_{h}$ symmetry, the ${ }^{5} \mathrm{D}$ state of a $\mathrm{d}^{6}$ $\mathrm{S}=2 \mathrm{Fe}(\mathrm{II})$ free ion splits into ${ }^{5} \mathrm{~T}_{2 \mathrm{~g}}$ ground and ${ }^{5} \mathrm{E}_{\mathrm{g}}$ excited states separated in energy by $10 \mathrm{Dq}$. An axial compression of the octahedron further splits the ${ }^{5} \mathrm{~T}_{2 \mathrm{~g}}$ degenerate set into a ${ }^{5} \mathrm{~B}_{2 \mathrm{~g}}$ ground state and a low-lying ${ }^{5} \mathrm{E}_{\mathrm{g}}$ excited state at an energy $\Delta$ above the ground state. This corresponds to a positive ZFS, $+\mathrm{D}$. Here, the lower- and higher-energy ${ }^{5} \mathrm{E}_{\mathrm{g}}$ excited states will be referred to as ${ }^{5} E_{g}(1 a, 1 b)$ and ${ }^{5} E_{g}(2 a, 2 b)$, respectively. The axial distortion can also split the ${ }^{5} \mathrm{E}_{\mathrm{g}}(2 \mathrm{a}, 2 \mathrm{~b})$ excited states into ${ }^{5} \mathrm{~A}_{1 \mathrm{~g}}$ and ${ }^{5} \mathrm{~B}_{2 \mathrm{~g}}$ states separated in energy by $\Delta^{5} E_{g}$. For $-D$, the overall splittings are reversed, and the

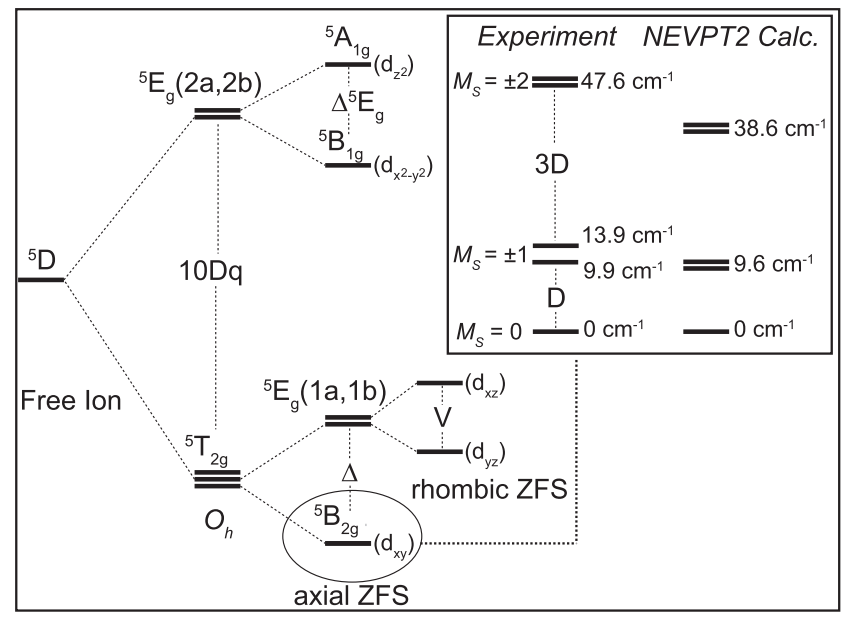

FIG. 1. State splitting diagram for a $d^{6} S=2$ transition metal complex. (Inset) Experimental $M_{S}$ sublevel splittings for $\mathrm{FeSiF}_{6} \cdot 6 \mathrm{H}_{2} \mathrm{O}$, $\left[\mathrm{Fe}\left(\mathrm{H}_{2} \mathrm{O}\right)_{6}\right]^{2+}$, from electronic Raman spectroscopy ${ }^{33}$ compared to the complete active space self-consistent field/N-electron valence state second order perturbation theory (CASSCF/NEVPT2) calculated energy levels.
${ }^{5} E_{g}(1 a, 1 b)$ is an orbitally degenerate ground state. Note that only the $+\mathrm{D}$ scenario is considered here. An additional rhombic distortion can split the ${ }^{5} \mathrm{E}_{\mathrm{g}}(1 \mathrm{a}, 1 \mathrm{~b})$ excited states by an energy $\mathrm{V}$. While the $\mathrm{S}=2$ ${ }^{5} \mathrm{~B}_{2 \mathrm{~g}}$ ground state is orbitally nondegenerate, it is fivefold degenerate in spin $\left(M_{S}\right)$. The degeneracy of the five $M_{S}$ sublevels deriving from the many-electron molecular ${ }^{5} \mathrm{~B}_{2 \mathrm{~g}}$ ground state can split in the absence of a magnetic field due to excited state SOC (inset, Fig. 1). Through perturbation theory and taking into account second order SOC, D can be expressed as follows: ${ }^{3}$

$$
D=\frac{\lambda^{2} \eta}{\Delta}
$$

where $\lambda=-\zeta / 2 S$ (with $\lambda$ being the many-electron SOC parameter, $\zeta$ being the metal-based one-electron SOC constant, and S being the total electron spin; the negative sign is taken for a greater than half-filled electronic configuration), $\Delta$ is the energy weighted average of the ${ }^{5} E_{g}(1 a, 1 b)$ excited states above the ground state, and $\eta$ is a parameter that accounts for the covalent reduction of $\zeta$. For $S=2$ $\mathrm{Fe}(\mathrm{II}), \zeta=400 \mathrm{~cm}^{-1}$, and thus $\lambda=-100 \mathrm{~cm}^{-1}$.

In order to identify and understand the nature of spin-phonon coupling, we evaluate the change in the ZFS along specific normal modes of vibration, $Q_{i}$. Note that the model described here is applicable to both phonons and vibrations; thus, we simply use the terminology of spin-phonon coupling as a general description. Taking the partial derivative of Eq. (1) with respect to a vibrational coordinate $Q_{i}$ gives

$$
\partial \mathrm{D} / \partial Q_{i}=\lambda^{2} \frac{\Delta\left(\partial \eta / \partial Q_{i}\right)-\eta\left(\partial \Delta / \partial Q_{i}\right)}{\Delta^{2}} .
$$

This derivative expression is referred to here as a linear spin-phonon coupling term. In the regime where $\eta\left(\partial \Delta / \partial Q_{i}\right) \gg$ $\Delta\left(\partial \eta / \partial Q_{i}\right)$, Eq. (2) simplifies to

$$
\partial \mathrm{D} / \partial Q_{i}=-\frac{\lambda^{2} \eta\left(\partial \Delta / \partial Q_{i}\right)}{\Delta^{2}} .
$$

Thus, in this regime, the linear spin-phonon coupling term is predicted to have an inverse square dependence on $\Delta$ and a linear dependence on the partial derivative of the excited state energy, $\partial \Delta \partial Q_{i}$, which is directly related to the so-called linear coupling term. $^{34}$ To distinguish this term from the spin-phonon coupling term, which deals solely with the axial ZFS, D, the linear coupling term dealing with the partial derivatives of excited state energies will be referred to as a linear excited state coupling term. This term, effectively represented by the slope of the excited state PES at the nuclear coordinates of the ground state geometry, is given by ${ }^{34}$

$$
E_{\text {elec }-n u c}^{e}=\left\langle\psi_{e}^{\text {elec }}\left|\left(\frac{\partial \boldsymbol{H}_{L F}}{\partial Q_{i}}\right)_{0}\right| \psi_{e}^{\text {elec }}\right\rangle Q_{i} .
$$

In addition to evaluating Eq. (4) at the ground state equilibrium geometry, we also evaluate the linear excited state coupling term along the ground state PES. In those cases, the linear excited state coupling term is normalized to the slope in the ground state PES.

When $\eta\left(\partial \Delta / \partial Q_{i}\right) \ll \Delta\left(\partial \eta / \partial Q_{i}\right)$, Eq. (2) simplifies to

$$
\partial \mathrm{D} / \partial Q_{i}=\frac{\lambda^{2}\left(\partial \eta / \partial Q_{i}\right)}{\Delta},
$$


and $\partial D / \partial Q_{i}$ will have an inverse dependence on $\Delta$ and a linear dependence on the partial derivative of covalency, $\eta$. However, as shown below, the complexes considered here are quite ionic and are largely in the regime where Eq. (3) best describes the linear spin-phonon coupling terms. Here, our aim is to emphasize the importance of the excited state energies and their linear excited state coupling terms [Eq. (4)] for understanding the linear spin-phonon coupling terms [Eq. (2)], as they both play key roles in understanding spin-phonon coupling. Thus, from the abovementioned considerations, the higher the energy separation between the ${ }^{5} \mathrm{E}_{\mathrm{g}}(1 \mathrm{a}, 1 \mathrm{~b})$ excited states and the ${ }^{5} \mathrm{~B}_{2 \mathrm{~g}}$ ground state, the lower the $\partial \mathrm{D} / \partial Q_{i}$ term by at least an inverse dependence.

To analyze the mechanism of ISC in Sec. II D 2, it will be useful to consider the ratio of linear spin-phonon coupling terms for two different excited states within the same molecule. Using Eq. (3), this ratio is

$$
\left(\partial \mathrm{D}_{E S 1} / \partial Q_{i}\right) /\left(\partial \mathrm{D}_{E S 2} / \partial Q_{i}\right)=n \cdot\left[\frac{\left(\Delta_{E S 2}\right)^{2}}{\left(\Delta_{E S 1}\right)^{2}}\right] \cdot\left[\frac{\left(\partial \Delta_{E S 1} / \partial Q_{i}\right)}{\left(\partial \Delta_{E S 2} / \partial Q_{i}\right)}\right],
$$

where $\Delta_{\mathrm{ES} 1}$ and $\Delta_{\mathrm{ES} 2}$ are the energies of two different excited states above the ground state, which each contribute to the overall ground state ZFS of a given transition metal complex [e.g., the ${ }^{5} \mathrm{E}_{\mathrm{g}}(1 \mathrm{a}, 1 \mathrm{~b})$ excited states, the lowest energy ${ }^{3} \mathrm{~T}_{1 \mathrm{~g}}$ excited states, and the ${ }^{5} \mathrm{~B}_{2 \mathrm{~g}}$ ground state considered here], and $n$ is a scaling factor accounting for differences in ZFS contributions from ES1 and ES2. Equation (6), thus, determines the individual roles of intramolecular linear excited state and spin-phonon coupling terms to the mechanism of ISC. As shown in Sec. II D 2, for the complex considered here, Eq. (6) results in a pseudo-exponential variation of the ratio of linear spin-phonon coupling terms, which is an important result for understanding spin-phonon coupling and dynamic ZFS contributions to spin conversion processes in transition metal complexes.

As exemplified in our recent work on evaluating spin-phonon coupling terms in transition metal qubit candidates, the parameters in the model described here are directly related to spectroscopic observables and calculable quantities. Furthermore, while this treatment is generated for $O_{h}$ symmetry and distorted $\mathrm{S}=2 \mathrm{Fe}$ (II) complexes, it can be performed analogously for any $\mathrm{S}>\frac{1}{2}$ transition metal complex. Finally, the terms calculated from the equations mentioned above will be sensitive to the absolute values of the excited state energies. Thus, a spectroscopically calibrated computational methodology is important for obtaining more quantitative insights. Below, a multireference $a b$ initio approach [i.e., complete active space self-consistent field (CASSCF) N-electron valence state second order perturbation theory (NEVPT2)] is utilized to benchmark to a well-studied $\mathrm{Fe}(\mathrm{II})$ complex $\left(\left[\mathrm{Fe}\left(\mathrm{H}_{2} \mathrm{O}\right)_{6}\right]^{2+}\right)$ with an experimentally known axial ZFS parameter and partially defined spinallowed and spin-forbidden excited state manifold. Similar $a b$ initio approaches have been applied with great success to the calculation of spin Hamiltonian parameters of transition metal complexes, ${ }^{6,735-37}$ including SCO complexes. ${ }^{38-42}$ Once the methodology is applied to $\left[\mathrm{Fe}\left(\mathrm{H}_{2} \mathrm{O}\right)_{6}\right]^{2+}$, it is then extended to additional complexes for the evaluation of linear spin-phonon [Eq. (2)] and excited state coupling [Eq. (4)] terms.

\section{B. Geometric contributions to the ground and excited state electronic structures of several Fe(II) complexes}

In this section, detailed $a b$ initio calculations have been carried out on four different six-coordinate high-spin $\mathrm{Fe}(\mathrm{II})$ structures, two each for $\left[\mathrm{Fe}\left(\mathrm{H}_{2} \mathrm{O}\right)_{6}\right]^{2+}$ and $\left[\mathrm{Fe}\left(\mathrm{NH}_{3}\right)_{6}\right]^{2+}$. For both complexes, these geometries consist of a symmetric structure [all Fe(II)-L bond distances equal] obtained from neutron diffraction (for $\left.\left[\mathrm{Fe}\left(\mathrm{H}_{2} \mathrm{O}\right)_{6}\right]^{2+}\right)^{43}$ or $\mathrm{x}$-ray crystallography (for $\left.\left[\mathrm{Fe}\left(\mathrm{NH}_{3}\right)_{6}\right]^{2+}\right),{ }^{44-46}$ as well as BP86 density functional theory (DFT) optimized structures. As discussed in this section and Sec. II C, the ground state equilibrium geometry plays an important role in determining the energy splittings of the states given in Fig. $1\left[{ }^{5} \mathrm{E}_{\mathrm{g}}(1 \mathrm{a}, 1 \mathrm{~b})\right.$ in particular], which has a large contribution to the ground state ZFS. The comparisons between the symmetric and optimized structures allow for a quantitative evaluation of geometric contributions to the static and dynamic electronic structures of simple $\mathrm{Fe}(\mathrm{II})$ coordination complexes and, ultimately, the spin-phonon coupling terms. While the magnetic ground state of $\left[\mathrm{Fe}\left(\mathrm{H}_{2} \mathrm{O}\right)_{6}\right]^{2+}$ has been much more thoroughly characterized, the model outlined in this study is currently being extended to Fe(II)-based SCO complexes and photosensitizers, which often feature an $\mathrm{Fe}(\mathrm{II}) \mathrm{N}_{6}$ coordination sphere. Therefore, $\left[\mathrm{Fe}\left(\mathrm{NH}_{3}\right)_{6}\right]^{2+}$ provides an ideal initial comparison, and the overall good agreement between experiment and the CASSCF/NEVPT2 calculations for $\left[\mathrm{Fe}\left(\mathrm{H}_{2} \mathrm{O}\right)_{6}\right]^{2+}$ allows for a more confident extension to other simple $\mathrm{Fe}(\mathrm{II})$ coordination complexes. Finally, while water ligands can potentially result in anisotropic bonding contributions with the d-orbitals from $\mathrm{a}_{1}$ and $b_{1}$ donor molecular orbitals of $\mathrm{H}_{2} \mathrm{O}, \mathrm{NH}_{3}$ is a $\sigma$-donor only ligand and can be used to further simplify the spin-phonon coupling analyses.

The ground and excited state electronic structure of the high spin $\mathrm{S}=2$ ferrous fluorosilicate (FFS, $\mathrm{FeSiF}_{6} \cdot 6 \mathrm{H}_{2} \mathrm{O},\left[\mathrm{Fe}\left(\mathrm{H}_{2} \mathrm{O}\right)_{6}\right]^{2+}$ ) has been elucidated using a variety of spectroscopic methods. Magnetic susceptibility gives $\mathrm{D}=+10.9 \mathrm{~cm}^{-1}$, which is similar to that obtained using far-infrared absorption spectroscopy $(\mathrm{D}=+11.9$ and $\left.|\mathrm{E}|=0.67 \mathrm{~cm}^{-1}\right){ }^{48,49}$ Electronic Raman has resolved the individual $M_{S}= \pm 1$ sublevels at $9.9 \pm 0.2 \mathrm{~cm}^{-1}$ and $13.9 \pm 0.2 \mathrm{~cm}^{-1}$ and the $M_{S}= \pm 2$ sublevels at $47.6 \pm 0.2 \mathrm{~cm}^{-1}$ above the $M_{S}=0$ level (Fig. 1, inset). ${ }^{33}$ Excited state spectroscopies, including electronic absorption $^{50}$ and magnetic circular dichroism (MCD), ${ }^{51}$ placed the split ${ }^{5} \mathrm{E}_{\mathrm{g}}$ excited state at $9600 \mathrm{~cm}^{-1}$ and $10800 \mathrm{~cm}^{-1}$ and a spin-forbidden transition (likely the lowest energy ${ }^{3} \mathrm{~T}_{1 \mathrm{~g}}$ ) at $\sim 13700 \mathrm{~cm}^{-1}$. These data for $\left[\mathrm{Fe}\left(\mathrm{H}_{2} \mathrm{O}\right)_{6}\right]^{2+}$ can be used to evaluate CASSCF/NEVPT2 calculations of the zero-field split $M_{S}$ sublevels of the orbitally nondegenerate ${ }^{5} \mathrm{~B}_{2 \mathrm{~g}}$ ground state and the associate spin-allowed and spin-forbidden excited state energies.

The neutron diffraction structure of FFS corresponds to an octahedron with $\mathrm{Fe}-\mathrm{O}$ bond lengths of $2.14 \AA^{43}{ }^{43}$ Note that subsequent spectroscopic analyses support a structure that is slightly compressed along the $\mathrm{z}$-axis, ${ }^{52}$ which is more consistent with the structure obtained from DFT geometry optimization (vide infra). Nonetheless, as described below, we find it insightful to compare symmetric structures to geometry optimized structures. With the neutron diffraction structure and a five $3 \mathrm{~d}$ orbital and six electron active space with 5 quintets, 17 triplets, and 15 singlets, the axial ZFS parameter calculated using the ORCA ${ }^{53,54}$ program is $9.6 \mathrm{~cm}^{-1}$, 
TABLE I. Summary of CASSCF/NEVPT2 calculated energies for $\left[\mathrm{Fe}\left(\mathrm{H}_{2} \mathrm{O}\right)_{6}\right]^{2+}$ and $\left[\mathrm{Fe}\left(\mathrm{NH}_{3}\right)_{6}\right]^{2+}$

\begin{tabular}{lrccccc}
\hline \hline Structure & $D^{\mathrm{a}}$ & $|\mathrm{E} / \mathrm{D}|$ & $M_{s}= \pm 1, \pm 2^{\mathrm{a}}$ & ${ }^{5} \mathrm{E}_{\mathrm{g}}(1 \mathrm{a}, 1 \mathrm{~b})^{\mathrm{a}}$ & ${ }^{5} \mathrm{E}_{\mathrm{g}}(2 \mathrm{a}, 2 \mathrm{~b})^{\mathrm{a}}$ & ${ }^{3} \mathrm{~T}_{1 \mathrm{~g}}{ }^{\mathrm{a}}$ \\
\hline$\left[\mathrm{Fe}\left(\mathrm{H}_{2} \mathrm{O}\right)_{6}\right]^{2+\mathrm{b}}$ & 9.6 & 0.003 & $9.6 / 9.6,38.6 / 38.6$ & 2100 & 10135 & 15440 \\
{$\left[\mathrm{Fe}\left(\mathrm{H}_{2} \mathrm{O}\right)_{6}\right]^{2+c}$} & 18.3 & 0.221 & $6.0 / 30.3,73.6 / 77.7$ & 635 & 8420 & 18530 \\
{$\left[\mathrm{Fe}\left(\mathrm{NH}_{3}\right)_{6}\right]^{2+\mathrm{d}}$} & 37.9 & 0.101 & $8.5 / 28.0,139.5 / 147.2$ & 195 & 11250 & 11380 \\
{$\left[\mathrm{Fe}\left(\mathrm{NH}_{3}\right)_{6}\right]^{2+c}$} & 38.4 & 0.072 & $9.8 / 25.2,141 / 147$ & 185 & 9425 & 11640 \\
\hline \hline
\end{tabular}

${ }^{\mathrm{a}} \mathrm{cm}^{-1}$.

${ }^{\mathrm{b}}$ Neutron diffraction structure.

${ }^{\mathrm{c}} \mathrm{BP} 86$ optimized structure.

${ }^{\mathrm{d}} \mathrm{X}$-ray idealized structure.

which is in good agreement with experiment. The energies of the four lowest-energy $M_{S}$ sublevels can also be obtained directly from the SOC corrected absorption spectrum; for this structure, they are $9.6 \mathrm{~cm}^{-1}, 9.6 \mathrm{~cm}^{-1}, 38.6 \mathrm{~cm}^{-1}$, and $38.6 \mathrm{~cm}^{-1}$, again in fairly good agreement with experiment (Fig. 1, inset). Additional benchmarking calculations were carried out to test the robustness of the active space and number of states included in the calculation. The computed results are not particularly sensitive to additional active space bonding orbitals and states (Table S1).

In addition to the zero-field split $M_{S}$ sublevels, the ${ }^{5} \mathrm{E}_{\mathrm{g}}(2 \mathrm{a}, 2 \mathrm{~b})$ excited state energies were calculated to be $10135 \mathrm{~cm}^{-1}$, which is in good agreement with experiment (average of $10200 \mathrm{~cm}^{-1}$ ). ${ }^{50,51}$ The lowest energy spin-forbidden triplet state $\left({ }^{3} \mathrm{~T}_{1 \mathrm{~g}}\right)$ is calculated to be at an average energy of $15440 \mathrm{~cm}^{-1}$. The lowest energy spin-forbidden singlet state, which, along with the ${ }^{3} \mathrm{~T}_{1 \mathrm{~g}}$, is of particular importance for ISC (vide infra), is calculated to be at an energy of $18530 \mathrm{~cm}^{-1}$. The ${ }^{5} \mathrm{E}_{\mathrm{g}}(1 \mathrm{a}, 1 \mathrm{~b})$ components (from the parent ${ }^{5} \mathrm{~T}_{2 \mathrm{~g}}$ ground state) are both calculated to be at an average energy of $2100 \mathrm{~cm}^{-1}$. As discussed above, the ZFS of the ${ }^{5} \mathrm{~B}_{2 \mathrm{~g}}$ component largely derives from the SOC of these ${ }^{5} E_{g}(1 a, 1 b)$ excited states. Accordingly, ORCA provides an estimate of the individual excited state contributions to the value of D. The ${ }^{5} \mathrm{E}_{\mathrm{g}}(\mathrm{la}, 1 \mathrm{~b})$ states are predicted to contribute $\sim 5.0 \mathrm{~cm}^{-1}$, while the lowest energy triplet state $\left({ }^{3} \mathrm{~T}_{1 \mathrm{~g}}\right.$ in $\left.\mathrm{O}_{h}\right)$ is estimated to contribute $\sim 1.8 \mathrm{~cm}^{-1}$. Note that all calculated values given here and below from Sec. II B are summarized in Table I.

In addition to the experimental structure, a full geometry optimization using the BP86 functional was carried out starting from the neutron diffraction structure of $\left[\mathrm{Fe}\left(\mathrm{H}_{2} \mathrm{O}\right)_{6}\right]^{2+}$. Notably, the BP86 optimized structure features a slight axial compression along the $\mathrm{z}$ axis (2.13 $\AA$ vs $2.14 \AA)$ and a slight elongation of the equatorial $\mathrm{Fe}-\mathrm{O}$ bonds ( $2.16 \AA$ vs $2.14 \AA$ ). Note that this distortion corresponds to the $e_{g}(\theta)$ mode given in Fig. 2. Using the optimized structure, the axial ZFS is calculated to be $18.3 \mathrm{~cm}^{-1}$, increased relative to $9.6 \mathrm{~cm}^{-1}$ for the neutron diffraction structure. The $M_{S}$ sublevels from the SOC corrected absorption spectrum are at $6.0 \mathrm{~cm}^{-1}, 30.3 \mathrm{~cm}^{-1}, 73.6 \mathrm{~cm}^{-1}$, and $77.7 \mathrm{~cm}^{-1}$. The larger and smaller magnitudes of the splittings of the $M_{S}= \pm 1$ and \pm 2 sublevels, respectively, are qualitatively consistent with experiment (Fig. 1, inset). The calculated ${ }^{5} \mathrm{E}_{\mathrm{g}}(2 \mathrm{a}, 2 \mathrm{~b})$ and ${ }^{5} \mathrm{E}_{\mathrm{g}}(\mathrm{la}, 1 \mathrm{~b})$ states are at $8065 / 8770 \mathrm{~cm}^{-1}$ and $545 / 720 \mathrm{~cm}^{-1}$, respectively, while the triplet and singlet states are $15140 \mathrm{~cm}^{-1}$ and $18845 \mathrm{~cm}^{-1}$, respectively. Overall, the increased ZFS for this structure relative to the neutron diffraction structure is consistent with lower energy ${ }^{5} \mathrm{E}_{\mathrm{g}}(1 \mathrm{a}, 1 \mathrm{~b})$ excited states, which contribute $\sim 15.8 \mathrm{~cm}^{-1}$ to the total axial ZFS, with a small $\sim 0.8 \mathrm{~cm}^{-1}$ contribution from the lowest energy triplet. Thus, even the slight geometric distortion along the $e_{g}(\theta)$ mode due to geometry optimization has a large effect on the ground state ZFS and the energies of the ${ }^{5} \mathrm{E}_{\mathrm{g}}(1 \mathrm{a}, 1 \mathrm{~b})$ excited states.

As described above, an extension to $\left[\mathrm{Fe}\left(\mathrm{NH}_{3}\right)_{6}\right]^{2+}$ provides a direct comparison to $\left[\mathrm{Fe}\left(\mathrm{H}_{2} \mathrm{O}\right)_{6}\right]^{2+}$, as well as an initial evaluation of $\mathrm{Fe}(\mathrm{II}) \mathrm{N}_{6}$ coordination complexes. Experimental structures for $\left[\mathrm{Fe}\left(\mathrm{NH}_{3}\right)_{6}\right]^{2+}$ have been reported with $\mathrm{Fe}-\mathrm{N}$ bond distances varying from $\sim 2.21 \AA$ to $2.23 \AA$ depending on the counterion. ${ }^{44-46}$ Thus, we used a semi-idealized structure here, which has $\mathrm{Fe}-\mathrm{N}$ bond distances of $2.22 \AA$ A. For this structure, the axial ZFS of $\left[\mathrm{Fe}\left(\mathrm{NH}_{3}\right)_{6}\right]^{2+}$ is calculated to be significantly larger than the neutron diffraction $\left[\mathrm{Fe}\left(\mathrm{H}_{2} \mathrm{O}\right)_{6}\right]^{2+}$ structure $\left(37.9 \mathrm{~cm}^{-1}\right.$ vs $\left.9.6 \mathrm{~cm}^{-1}\right)$. The $M_{S}$ sublevels are at $8.5 \mathrm{~cm}^{-1}, 28.0 \mathrm{~cm}^{-1}, 139.5 \mathrm{~cm}^{-1}$, and $147.2 \mathrm{~cm}^{-1}$. Experimentally, bands at $12400 \mathrm{~cm}^{-1}$ and $9000 \mathrm{~cm}^{-1}$ have been assigned to components of the ${ }^{5} \mathrm{~T}_{2 \mathrm{~g}} \rightarrow{ }^{5} \mathrm{E}_{\mathrm{g}}(2 \mathrm{a}, 2 \mathrm{~b})$ transition. ${ }^{55}$ Here, for the
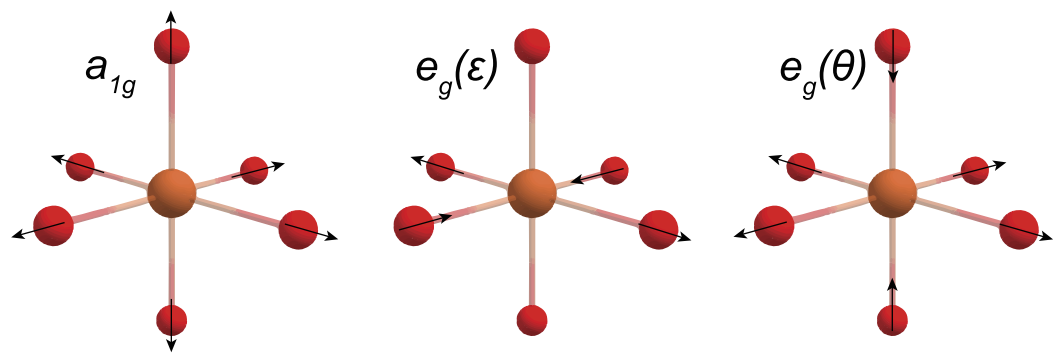

FIG. 2. Three key $\mathrm{ML}_{6} \mathrm{O}_{h}$ normal modes. 
$\mathrm{x}$-ray idealized structure, the ${ }^{5} \mathrm{E}_{\mathrm{g}}(2 \mathrm{a}, 2 \mathrm{~b})$ and ${ }^{5} \mathrm{E}_{\mathrm{g}}(1 \mathrm{a}, 1 \mathrm{~b})$ states are calculated at $11250 / 11255 \mathrm{~cm}^{-1}$ and $145 / 240 \mathrm{~cm}^{-1}$, respectively, while the lowest energy triplet and singlet states are $11380 \mathrm{~cm}^{-1}$ and $11640 \mathrm{~cm}^{-1}$, respectively. The relatively large ZFS for the $\mathrm{x}-$ ray idealized structure of $\left[\mathrm{Fe}\left(\mathrm{NH}_{3}\right)_{6}\right]^{2+}$ is consistent with the low energies of the ${ }^{5} \mathrm{E}_{\mathrm{g}}(1 \mathrm{a}, 1 \mathrm{~b})$ states, which contribute $\sim 31.6 \mathrm{~cm}^{-1}$ to the total axial ZFS, with a small $\sim 1.6 \mathrm{~cm}^{-1}$ contribution from the lowest energy triplet.

As for $\left[\mathrm{Fe}\left(\mathrm{H}_{2} \mathrm{O}\right)_{6}\right]^{2+}$, a BP86 fully optimized structure was also obtained for $\left[\mathrm{Fe}\left(\mathrm{NH}_{3}\right)_{6}\right]^{2+}$. Upon optimization, the $\mathrm{Fe}-\mathrm{N}$ bonds expand to 2.31/2.30 $\AA$ and 2.28/2.28/2.29/2.29 $\AA$ in the axial and equatorial planes, respectively. The computed axial ZFS for the optimized structure is slightly larger than that of the idealized structure $\left(38.4 \mathrm{~cm}^{-1}\right.$ vs $37.9 \mathrm{~cm}^{-1}$, respectively), with $\sim 32.4$ and $\sim 1.1 \mathrm{~cm}^{-1}$ of the ZFS deriving from the ${ }^{5} \mathrm{E}_{\mathrm{g}}(1 \mathrm{a}, 1 \mathrm{~b})$ and ${ }^{3} \mathrm{~T}_{1 \mathrm{~g}}$ states, respectively. The $M_{S}$ sublevels are at $9.8 \mathrm{~cm}^{-1}, 25.2 \mathrm{~cm}^{-1}, 141.0 \mathrm{~cm}^{-1}$, and $147.0 \mathrm{~cm}^{-1}$. The ${ }^{5} \mathrm{E}_{\mathrm{g}}(2 \mathrm{a}, 2 \mathrm{~b})$ and ${ }^{5} \mathrm{E}_{\mathrm{g}}(1 \mathrm{a}, 1 \mathrm{~b})$ states are predicted at $9235 / 9615 \mathrm{~cm}^{-1}$ and $155 / 210 \mathrm{~cm}^{-1}$, respectively, while the triplet and singlet states are $13140 \mathrm{~cm}^{-1}$ and $15220 \mathrm{~cm}^{-1}$, respectively. Overall, upon optimization and expansion of the $\mathrm{Fe}-\mathrm{N}$ bonds, the energies of the ${ }^{5} \mathrm{E}_{\mathrm{g}}(1 \mathrm{a}, 1 \mathrm{~b})$ states do not change as dramatically as the ${ }^{5} E_{g}(2 a, 2 b)$ states, which is consistent with similar computed axial ZFS values between the two structures.
In summary, as given in Table I, slight geometric distortions along the $e_{g}(\theta)$ mode for the DFT optimized structure relative to the symmetric $\left[\mathrm{Fe}\left(\mathrm{H}_{2} \mathrm{O}\right)_{6}\right]^{2+}$ structure lead to a significant change in the calculated axial ZFS $\left(9.6 \mathrm{~cm}^{-1}\right.$ vs $\left.18.3 \mathrm{~cm}^{-1}\right)$. This change derives from the decrease in the energies of the ${ }^{5} \mathrm{E}_{\mathrm{g}}(1 \mathrm{a}, 1 \mathrm{~b})$ excited states upon geometric distortion. Conversely, for $\left[\mathrm{Fe}\left(\mathrm{NH}_{3}\right)_{6}\right]^{2+}$, geometric distortion upon geometry optimization leads to only slight changes in the axial ZFS $\left(37.9 \mathrm{~cm}^{-1}\right.$ vs $\left.38.4 \mathrm{~cm}^{-1}\right)$, which is more consistent with the small deviations in the ${ }^{5} \mathrm{E}_{\mathrm{g}}(1 \mathrm{a}, 1 \mathrm{~b})$ excited states for the symmetric and optimized structures $\left(195 \mathrm{~cm}^{-1}\right.$ vs $185 \mathrm{~cm}^{-1}$, respectively).

\section{Spin-phonon coupling terms}

In the three subsections of Sec. II C, the four Fe(II) structures considered above are first used in Sec. II C 1 to test the geometric structural dependence on the nature and magnitudes of the calculated linear spin-phonon and excited state coupling terms. These are found to differ significantly for different normal modes of a complex and depend strongly on the structure of the equilibrium geometry. These differences in linear spin-phonon coupling terms [Eq. (2)] derive from differences in their corresponding linear excited state coupling terms [Eq. (4)] (Fig. 3). Second, in Sec. II C 2,
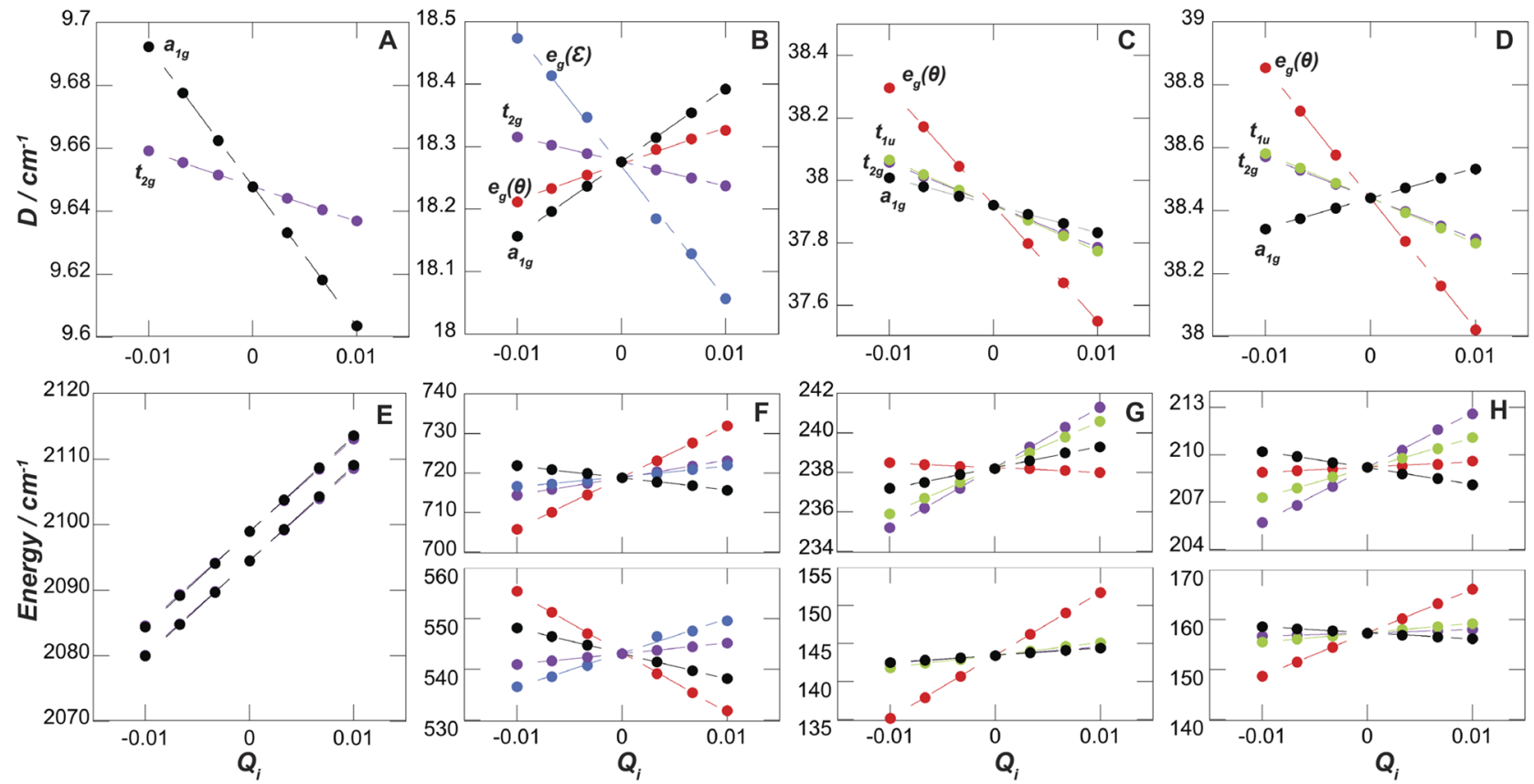

FIG. 3. Linear spin-phonon coupling term $\left[D\left(\mathrm{~cm}^{-1}\right)\right.$ vs $\left.Q_{i}\right]$ (a)-(d) and excited state coupling term $\left[{ }^{5} E_{g}(1 \mathrm{a}, 1 \mathrm{~b})\left(\mathrm{cm}^{-1}\right)\right.$ vs $\left.Q_{i}\right]$ (e)-(h) calculations for the neutron diffraction $(a)$ and $(\mathrm{e})$ and $\mathrm{BP} 86$ optimized structures $(\mathrm{b})$ and (f) of $\left[\mathrm{Fe}\left(\mathrm{H}_{2} \mathrm{O}\right)_{6}\right]^{2+}$ and the $\mathrm{x}$-ray crystallographic-based $(\mathrm{c})$ and $(\mathrm{g})$ and $\mathrm{BP} 86$ optimized structures $(\mathrm{d})$ and $(\mathrm{h})$ of $\left[\mathrm{Fe}(\mathrm{NH})_{6}\right]^{2+}$ For the neutron diffraction structure of $\left[\mathrm{Fe}\left(\mathrm{H}_{2} \mathrm{O}\right)_{6}\right]^{2+}$ [panel (e)], the ${ }^{5} \mathrm{E}_{g}(1 \mathrm{a}, 1 \mathrm{~b})$ excited states are close in energy and only one panel is given. For other structures, the ${ }^{5} E_{g}(1 a, 1 b)$ excited states are split at the equilibrium geometry, and panels $(f)-(h)$ are divided into two subsections for visualization of both excited state components. Specific normal mode assignments are given as $a_{1 g}$ (black), $e_{g}(\varepsilon)$ (blue), $e_{g}(\theta)$ (red), a $t_{2 g}$ mode (purple), and a $t_{1 u}$ mode (green). The sign of the slopes are not of particular importance here. However, the slopes for the linear spin-phonon coupling terms are inverted relative to the corresponding linear excited state coupling term slopes, reflecting the inverse dependence of $D$ on the ${ }^{5} E_{g}(1 a, 1 b)$ excited state energies. 
quantitative comparisons between the linear spin-phonon coupling terms for different structures are made, wherein key differences between structures are linked to the relative energies of the ${ }^{5} E_{g}(1 a, 1 b)$ states in Fig. 1, highlighting important electronic structure contributions to the validity of the right-hand sides (RHSs) of Eqs. (2) and (3). These observations in Sec. II C 2 are further expanded upon in Sec. II C 3, where it is demonstrated that the presence of in-state orbital angular momentum can lead to deviations between linear spin-phonon coupling terms calculated using the left-hand or right-hand sides of Eqs. (2) and (3). Together, these sections provide a detailed description of the combined geometric and electronic structural contributions to the nature of spin-phonon coupling in simple Fe(II) coordination complexes and motivate the extension of spin-phonon analyses to more extended regions of the ground and excited state PESs, including specific contributions to the mechanism of ISC (Sec. II D).

\section{Identifying coupling modes}

The 15 normal modes of vibration of an octahedron include $\Gamma_{\text {vib }}=a_{1 g}+e_{g}+t_{2 g}+2 t_{1 u}+t_{2 u}$. The $a_{1 g}, e_{g}$, and one $t_{1 u}$ set of modes are stretches and the other $t_{1 u}, t_{2 u}$, and $t_{2 g}$ modes are bends (Fig. S1). Calculated frequencies and distortion vectors for the neutron diffraction and BP86 optimized structures of $\left[\mathrm{Fe}\left(\mathrm{H}_{2} \mathrm{O}\right)_{6}\right]^{2+}$ are given in Tables $\mathrm{S} 2$ and $\mathrm{S} 3$, respectively. As mentioned above, the $\mathrm{Fe}-$ $\mathrm{O}$ bond distances for the experimental structure are all equivalent (2.14 $\mathrm{A})$, while the BP86 optimized structure features a slight compression along the $\mathrm{z}$-axis $(2.128 \AA)$ and elongation in the equatorial plane (2.161 $\AA$ and $2.166 \AA$ for $\mathrm{x}$-axis and y-axis, respectively). This distortion upon geometry optimization corresponds to the $e_{g}(\theta)$ component of the $e_{g}$ stretching vibrations (Fig. 2). For the neutron diffraction structure, there are 18 imaginary frequencies that correspond to water-based waging and rocking motions. However, all 15 modes of the octahedron have positive frequencies up to $\sim 340 \mathrm{~cm}^{-1}$ (Table S2). For the purposes of this study, the absolute energies of the modes are not as important as the absolute atomic displacements, and the 15 modes considered here are all components of the normal modes of an octahedron.

The BP86 optimized structure represents a minimum energy structure with no imaginary frequencies. 26 normal modes are computed with energies $<400 \mathrm{~cm}^{-1}$. 15 of these modes are those of the octahedron (Table S3). The other 11 normal modes in this energy region correspond to water-based motions with little to no $\mathrm{M}-\mathrm{L}$ based motion. Below, we focus solely on M-L based bends and stretches.

Spin-phonon coupling terms were calculated as described in the Computational Methods section of the supplementary material. Briefly, to estimate the qualitative magnitudes of spin-phonon coupling terms, the axial ZFS parameter, D, was calculated along all normal modes of vibration [Figs. 3(a)-3(d) for the four structures considered here]. In addition to $D$, the energies of the ${ }^{5} E_{g}(1 a, 1 b)$ excited states were also followed [Figs. 3(e)-3(h)]. For the neutron diffraction structure, two of the 15 modes of the octahedron exhibit appreciable linear spin-phonon coupling terms. These are the totally symmetric stretch $\left[a_{1 g}\right.$, black markers in Figs. $3(\mathrm{a})$ and $\left.3(\mathrm{e})\right]$ and a component of a $t_{2 g}$ mode [purple markers in Figs. 3(a) and 3(e)]. The absolute values of the slopes are $4.4 \mathrm{~cm}^{-1}$ and $1.1 \mathrm{~cm}^{-1} / Q_{i}$ for the $a_{1 g}$ mode and the $t_{2 g}$ mode, respectively. For more quantitative analyses (vide infra), it is convenient to convert, when possible, the slope to an $\AA^{-1}$ scale. The absolute value of the slope for the $a_{1 g}$ mode is $18.5 \mathrm{~cm}^{-1} / \AA$.

For further analyses, we turn to the linear excited state coupling term introduced in Sec. II A [Eq. (4)]. This term is represented by the slope of the excited state PES at the nuclear coordinates of the ground state equilibrium geometry (i.e., the Franck-Condon point). When nonzero, a force is present on the excited state geometry, which will distort the structure along a normal mode to lower the total energy of the excited state. Thus, the linear excited state coupling term can be used to evaluate $\partial \Delta / \partial Q_{i}$ in Sec. II. Importantly, while the ZFS is a ground state property, it reflects contributions from excited state PESs and SOC contributions. Thus, modes that exhibit linear spin-phonon coupling terms [Eq. (2)] should exhibit appreciable linear excited state coupling terms [Eq. (4)]. Indeed, by comparing Figs. 3 (a) and $3(\mathrm{e})$, the $a_{1 g}$ and $t_{2 g}$ modes (black and purple markers, respectively) also exhibit corresponding linear excited state coupling terms. Modes that do not exhibit appreciable coupling are not shown in Fig. 3. However, for comparison and for all four structures considered here, all modes are given in Fig. S2. Also, fits to the data in Fig. 3 and Fig. S2 are provided in Tables S4-S11. Thus, from Figs. 3(a) and 3(d), the corresponding spin-phonon coupling terms and dynamic ground state ZFSs for a given vibrational mode can be directly linked to the corresponding dynamic contributions from distorted excited state PESs (i.e., excited state linear coupling terms).

The major ground state linear spin-phonon and excited state coupling terms for the BP86 optimized structure of $\left[\mathrm{Fe}\left(\mathrm{H}_{2} \mathrm{O}\right)_{6}\right]^{2+}$ are given in Figs. 3(b) and 3(f). Interestingly, additional modes exhibit linear coupling terms and become activated for this structure. In addition to the $a_{1 g}$ mode (black markers) and a $t_{2 g}$ mode (purple markers), both $e_{g}$ stretching modes exhibit appreciable slopes [i.e., $e_{g}(\theta)$, red markers, and $e_{g}(\varepsilon)$, blue markers]. Note that the vector displacements for the $e_{g}(\theta)$ and $e_{g}(\varepsilon)$ components are represented in Fig. 2. The axial ZFS slopes for the BP86 optimized structure of $\left[\mathrm{Fe}\left(\mathrm{H}_{2} \mathrm{O}\right)_{6}\right]^{2+}$ are $21.2 \mathrm{~cm}^{-1}, 11.8 \mathrm{~cm}^{-1}, 5.8 \mathrm{~cm}^{-1}$, and $3.9 \mathrm{~cm}^{-1} / Q_{i}$ for the $e_{g}(\varepsilon), a_{1 g}, e_{g}(\theta)$, and a $t_{2 g}$ mode, respectively.

The results for the neutron diffraction and BP86 optimized structures of $\left[\mathrm{Fe}\left(\mathrm{H}_{2} \mathrm{O}\right)_{6}\right]^{2+}$ provide a convenient comparison between coupling terms. For the neutron diffraction structure, the linear spin-phonon coupling term for the $a_{1 g}$ mode was $4.4 \mathrm{~cm}^{-1} / Q_{i}$, which equated to $18.5 \mathrm{~cm}^{-1} / \AA$. The linear spin-phonon coupling terms for the optimized structure were $11.8 \mathrm{~cm}^{-1} / Q_{i}$ or $50.7 \mathrm{~cm}^{-1} / \AA$, which is significantly larger than the neutron diffraction structure. The increased linear spin-phonon coupling term for the BP86 structure derives from the significantly lower energy ${ }^{5} \mathrm{E}_{\mathrm{g}}(1 \mathrm{a}, 1 \mathrm{~b})$ states $\left(545 / 720 \mathrm{~cm}^{-1}\right)$ relative to those for the neutron diffraction structure $\left(2095 / 2100 \mathrm{~cm}^{-1}\right)$, in accord with the model described in Sec. II A. Furthermore, the linear excited state coupling terms for the $e_{g}$ modes in the neutron diffraction structure exhibit no appreciable slope. This behavior is in distinct contrast to the appreciable linear excited state coupling terms for the BP86 structure [Fig. 3(f)]. Thus, by comparing structures of $\left[\mathrm{Fe}\left(\mathrm{H}_{2} \mathrm{O}\right)_{6}\right]^{2+}$, the presence of additional excited state linear coupling terms for the optimized structure gives rise to appreciable ground state linear spin-phonon coupling terms for additional modes. 
Calculations on the two different forms of $\left[\mathrm{Fe}\left(\mathrm{H}_{2} \mathrm{O}\right)_{6}\right]^{2+}$ were extended to two structures of $\left[\mathrm{Fe}\left(\mathrm{NH}_{3}\right)_{6}\right]^{2+}$. Similar to $\left[\mathrm{Fe}\left(\mathrm{H}_{2} \mathrm{O}\right)_{6}\right]^{2+}$, the first structure considered was derived from $\mathrm{x}$-ray crystal structures. There are several modes that exhibit linear spinphonon coupling terms. The absolute values of the largest slopes are $37.4 \mathrm{~cm}^{-1}, 14.6 \mathrm{~cm}^{-1}, 13.6 \mathrm{~cm}^{-1}$, and $8.7 \mathrm{~cm}^{-1} / Q_{i}$, which correspond to the $e_{g}(\theta)$ mode, a $t_{1 u}$ mode (green markers), a $t_{2 g}$ mode (purple markers), and the $a_{1 g}$ mode (black markers), respectively. The corresponding excited state linear coupling terms are given in Fig. $3(\mathrm{~g})$. For the $a_{1 g}$ mode, the linear spin-phonon coupling term equates to $41.6 \mathrm{~cm}^{-1} / \AA$. Again, the larger spin-phonon coupling term for the $a_{1 \mathrm{~g}}$ mode of this structure derives from the low energies of the ${ }^{5} \mathrm{E}_{\mathrm{g}}(1 \mathrm{a}, 1 \mathrm{~b})$ excited states $\left(145 / 240 \mathrm{~cm}^{-1}\right)$.

Spin-phonon coupling term calculations were finally extended to the optimized structure of $\left[\mathrm{Fe}\left(\mathrm{NH}_{3}\right)_{6}\right]^{2+}[\mathrm{Fig}$. 3(d)]. The absolute values of the largest slopes are $41.6 \mathrm{~cm}^{-1}, 14.2 \mathrm{~cm}^{-1}, 13.1 \mathrm{~cm}^{-1}$, and $9.6 \mathrm{~cm}^{-1} / Q_{i}$, which correspond to the $e_{g}(\theta)$ mode (red markers), a $t_{1 u}$ mode (green markers), a $t_{2 g}$ mode (purple markers), and the $a_{1 g}$ mode (black markers), respectively. The corresponding linear excited state coupling terms are given in Fig. $3(\mathrm{~h})$. For the $a_{1 g}$ mode, the spin-phonon coupling term equates to $45.3 \mathrm{~cm}^{-1} / \AA$. Again, the larger spin-phonon coupling for the $a_{1 g}$ mode of this structure term derives from the low energies of the ${ }^{5} \mathrm{E}_{\mathrm{g}}(1 \mathrm{a}, 1 \mathrm{~b})$ excited states $\left(155 / 210 \mathrm{~cm}^{-1}\right)$.

In summary, both linear spin-phonon [Eq. (2)] and excited state [Eq. (4)] coupling terms can be qualitatively evaluated for a given ground state equilibrium geometry of high-spin $\mathrm{Fe}(\mathrm{II})$ coordination complexes. In certain instances (e.g., the $a_{1 g}$ mode), the magnitude of the spin-phonon coupling terms can be put into a more quantitative $\AA^{-1}$ framework. It is shown that overall couplings are sensitive to the initial geometric structure, which results in significant mode-selective spin-phonon couplings across different structures. For example, the symmetric neutron diffraction structure of $\left[\mathrm{Fe}\left(\mathrm{H}_{2} \mathrm{O}\right)_{6}\right]^{2+}$ exhibits spin-phonon couplings with the $a_{1 g}$ mode and a $t_{2 g}$ mode. A slight distortion along the $e_{g}(\theta)$ mode upon geometry optimization activates mode couplings to both components of the $e_{g}$ set of vibrational modes. The mode-selective activation of linear spin-phonon coupling terms is a direct consequence of new linear excited state coupling terms in different structures. This result is of general importance for considering the roles of geometric and electronic structure on the nature of spin-phonon coupling in transition metal complexes.

\section{Quantitative spin-phonon coupling term comparisons}

With the ability to obtain linear spin-phonon and excited state coupling terms for the $a_{1 g}$ modes of multiple structures, direct comparisons can be made between the left-hand and right-hand sides of the expressions given in Sec. II. From Löwdin orbital population analyses, the $\mathrm{d} \pi$ and $\mathrm{d} \sigma$ orbital characters for $\left[\mathrm{Fe}\left(\mathrm{H}_{2} \mathrm{O}\right)_{6}\right]^{2+}$ and $\left[\mathrm{Fe}\left(\mathrm{NH}_{3}\right)_{6}\right]^{2+}$ structures are similar and $\sim 95 \%$, respectively. Thus, they are in the regime where Eq. (3) is appropriate for describing linear spin-phonon coupling terms. The first example will compare spin-phonon coupling terms calculated directly using the left-hand side of Eq. (3) to those obtained from the right-hand side for the neutron diffraction and BP86 optimized structures of $\left[\mathrm{Fe}\left(\mathrm{H}_{2} \mathrm{O}\right)_{6}\right]^{2+}$. For these, the linear spin-phonon coupling terms for the $a_{1 g}$ modes were calculated to be $18.5 \mathrm{~cm}^{-1} / \AA$ and $50.7 \mathrm{~cm}^{-1} / \AA$, respectively. This corresponds to a ratio of linear spin-phonon coupling terms of 0.365 . Using the right-hand side of Eq. (3) (equating $\lambda^{2} \eta$ for both structures), the calculated values of $\Delta$ [the energies of ${ }^{5} \mathrm{E}_{\mathrm{g}}(1 \mathrm{a}, 1 \mathrm{~b})$ excited states], and the linear excited state coupling terms [slopes of the ${ }^{5} \mathrm{E}_{\mathrm{g}}(1 \mathrm{a}, 1 \mathrm{~b})$ excited states], we estimate a ratio of linear spin-phonon coupling terms of 0.364 for the $a_{1 g}$ mode, in agreement to that calculated directly using the ratios of the linear spin-phonon coupling terms [i.e., the left-hand side of Eq. (3)]. Note that the value of 0.364 for the right-hand side of Eq. (3) is obtained using the average contributions from both ${ }^{5} \mathrm{E}_{\mathrm{g}}(1 \mathrm{a}, 1 \mathrm{~b})$ excited states [i.e., 0.190 and 0.538 for ${ }^{5} \mathrm{E}_{\mathrm{g}}(1 \mathrm{a})$ and ${ }^{5} \mathrm{E}_{\mathrm{g}}(1 \mathrm{~b})$ states, respectively] (Table II).

The same comparison can be extended to the $\mathrm{x}$-ray idealized and BP86 optimized structures of $\left[\mathrm{Fe}\left(\mathrm{NH}_{3}\right)_{6}\right]^{2+}$. The ratios of their linear spin-phonon coupling terms using the left-hand side of Eq. (3) relative to the neutron diffraction structure of $\left[\mathrm{Fe}\left(\mathrm{H}_{2} \mathrm{O}\right)_{6}\right]^{2+}$ are 0.445 and 0.408 , respectively. Using the right-hand side of Eq. (3), ratios of 0.108 and 0.090 are obtained for the $\mathrm{x}$-ray idealized and BP86 optimized structures of $\left[\mathrm{Fe}\left(\mathrm{NH}_{3}\right)_{6}\right]^{2+}$, respectively. Notably, for both $\left[\mathrm{Fe}\left(\mathrm{NH}_{3}\right)_{6}\right]^{2+}$ structures, the ratios obtained from the right-hand side of Eq. (3) differ significantly from the ratios obtained using the absolute values of the linear spin-phonon coupling terms [i.e., the left-hand side of Eq. (3)]. Importantly, given the significantly lower energies of the ${ }^{5} \mathrm{E}_{\mathrm{g}}(1 \mathrm{a}, 1 \mathrm{~b})$ excited states of the $\left[\mathrm{Fe}\left(\mathrm{NH}_{3}\right)_{6}\right]^{2+}$ structures relative to the $\left[\mathrm{Fe}\left(\mathrm{H}_{2} \mathrm{O}\right)_{6}\right]^{2+}$ structures (e.g., $143 / 238 \mathrm{~cm}^{-1}$ and $157 / 209 \mathrm{~cm}^{-1}$ for the $\mathrm{x}$-ray idealized and BP86 optimized structures of $\left[\mathrm{Fe}\left(\mathrm{NH}_{3}\right)_{6}\right]^{2+}$, respectively), these observations suggest a potentially important role of the absolute energies of the ${ }^{5} \mathrm{E}_{\mathrm{g}}(1 \mathrm{a}, 1 \mathrm{~b})$ excited states in the magnitudes of the linear spin-phonon coupling terms.

Finally, the analysis in this section has utilized 5 quintets, 15 triplets, and 17 singlets, respectively. The same analyses can be performed only considering three or five quintets to determine the extent to which other excited states can contribute to the linear spin-phonon coupling terms at the equilibrium geometries. Using only three quintet states, the ratio of spin-phonon coupling terms in the case for the $\left[\mathrm{Fe}\left(\mathrm{H}_{2} \mathrm{O}\right)_{6}\right]^{2+}$ structures, calculated using the lefthand side of Eq. (3), is 0.316 (middle entries in Table II). Using only five quintets, the ratio from the left-hand side is 0.405 . Thus, spin-forbidden excited states (triplets in this case) can play a role in the linear spin-phonon coupling terms evaluated using the lefthand side of Eq. (3). Generally, the relative contributions from spinallowed and spin-forbidden excited states to the linear spin-phonon coupling terms will depend on the initial relative contributions to the overall ZFS, as well as the magnitudes of the individual excited state linear coupling terms.

In summary, the linear spin-phonon coupling terms evaluated using the left-hand or right-hand sides of Eq. (3) are consistent for the $\left[\mathrm{Fe}\left(\mathrm{H}_{2} \mathrm{O}\right)_{6}\right]^{2+}$ structures. This observation suggests that dynamic covalency contributions to spin-phonon coupling terms are not particularly important for these structures. Furthermore, this demonstrates that the linear excited state coupling term contributions dominate the ground state linear spin-phonon coupling terms for $\left[\mathrm{Fe}\left(\mathrm{H}_{2} \mathrm{O}\right)_{6}\right]^{2+}$. Conversely, comparisons between $\left[\mathrm{Fe}\left(\mathrm{H}_{2} \mathrm{O}\right)_{6}\right]^{2+}$ and $\left[\mathrm{Fe}\left(\mathrm{NH}_{3}\right)_{6}\right]^{2+}$ point to important deviations between spin-phonon coupling terms obtained using the left-hand and right-hand sides of Eq. (3). These important deviations are evaluated in Sec. II C 3. 
TABLE II. Spin-phonon analyses for the $a_{1 g}$ mode of multiple Fe(II) complexes.

\begin{tabular}{|c|c|c|c|c|c|c|c|c|c|}
\hline Structure & $\mathrm{D}^{\mathrm{a}} \AA^{-1}$ & ${ }^{5} \mathrm{E}_{\mathrm{g}}(1 \mathrm{a}){ }^{\mathrm{a}}$ & ${ }^{5} E_{g}(1 b){ }^{a}$ & ${ }^{5} \mathrm{E}_{\mathrm{g}}(1 \mathrm{a}) \AA^{-1}$ & ${ }^{5} E_{g}(1 b) \AA^{-1}$ & Ratio $^{\mathrm{b}}$ & $\operatorname{Ratio}(1 a)^{c}$ & $\operatorname{Ratio}(1 b)^{c}$ & Ratio (ave) \\
\hline States & \multicolumn{9}{|c|}{5 quintets, 17 triplets, 15 singlets } \\
\hline$\left[\mathrm{Fe}\left(\mathrm{H}_{2} \mathrm{O}\right)_{6}\right]^{2+\mathrm{d}}$ & 18.5 & 2095 & 2100 & 6079 & 6095 & 1 & 1 & 1 & 1 \\
\hline$\left[\mathrm{Fe}\left(\mathrm{H}_{2} \mathrm{O}\right)_{6}\right]^{2+e}$ & 50.7 & 545 & 720 & 2147 & 1330 & 0.365 & 0.190 & 0.538 & 0.364 \\
\hline$\left[\mathrm{Fe}\left(\mathrm{NH}_{3}\right)_{6}\right]^{2+\mathrm{f}}$ & 41.6 & 145 & 240 & 460 & 510 & 0.445 & 0.062 & 0.154 & 0.108 \\
\hline$\left[\mathrm{Fe}\left(\mathrm{NH}_{3}\right)_{6}\right]^{2+\mathrm{e}}$ & 45.3 & 155 & 210 & 585 & 500 & 0.408 & 0.058 & 0.180 & 0.090 \\
\hline States & \multicolumn{9}{|c|}{3 quintets, 0 triplets, 0 singlets } \\
\hline$\left[\mathrm{Fe}\left(\mathrm{H}_{2} \mathrm{O}\right)_{6}\right]^{2+\mathrm{d}}$ & 17.8 & 2050 & 2050 & 5965 & 5950 & 1 & 1 & 1 & 1 \\
\hline$\left[\mathrm{Fe}\left(\mathrm{H}_{2} \mathrm{O}\right)_{6}\right]^{2+e}$ & 56.4 & 530 & 700 & 2125 & 1275 & 0.316 & 0.185 & 0.543 & 0.364 \\
\hline$\left[\mathrm{Fe}\left(\mathrm{NH}_{3}\right)_{6}\right]^{2+\mathrm{f}}$ & 39.0 & 150 & 245 & 455 & 470 & 0.456 & 0.069 & 0.180 & 0.125 \\
\hline$\left[\mathrm{Fe}\left(\mathrm{NH}_{3}\right)_{6}\right]^{2+e}$ & 43.2 & 160 & 205 & 595 & 410 & 0.412 & 0.060 & 0.146 & 0.103 \\
\hline States & \multicolumn{9}{|c|}{5 quintets, 0 triplets, 0 singlets } \\
\hline$\left[\mathrm{Fe}\left(\mathrm{H}_{2} \mathrm{O}\right)_{6}\right]^{2+\mathrm{d}}$ & 22.4 & 2030 & 2030 & 5915 & 5925 & 1 & 1 & 1 & 1 \\
\hline$\left[\mathrm{Fe}\left(\mathrm{H}_{2} \mathrm{O}\right)_{6}\right]^{2+e}$ & 55.3 & 520 & 690 & 2135 & 1290 & 0.405 & 0.182 & 0.528 & 0.355 \\
\hline$\left[\mathrm{Fe}\left(\mathrm{NH}_{3}\right)_{6}\right]^{2+\mathrm{f}}$ & 36.6 & 145 & 235 & 430 & 460 & 0.612 & 0.070 & 0.174 & 0.122 \\
\hline$\left[\mathrm{Fe}\left(\mathrm{NH}_{3}\right)_{6}\right]^{2+\mathrm{e}}$ & 40.1 & 155 & 200 & 570 & 400 & 0.559 & 0.060 & 0.140 & 0.100 \\
\hline
\end{tabular}

${ }^{\mathrm{a}} \mathrm{cm}^{-1}$.

${ }^{\mathrm{b}}$ From D/Å directly [left-hand side of Eq. (3)].

${ }^{c}$ From the right-hand side of Eq. (3).

${ }^{\mathrm{d}}$ Neutron diffraction structure.

${ }^{\mathrm{e}} \mathrm{BP} 86$ optimized structure.

${ }^{\mathrm{f}} \mathrm{X}$-ray idealized structure.

Finally, spin-forbidden excited states can play a role in determining the magnitude of the linear spin-phonon coupling terms. This reflects their relative overall percentage contributions to the ground state ZFS along specific normal modes and is expanded on in Sec. II D.

\section{Deviation of $D$ and spin-phonon coupling terms from model behavior}

As shown above, more quantitative analyses of spin-phonon and excited state coupling terms indicated that $\left[\mathrm{Fe}\left(\mathrm{NH}_{3}\right)_{6}\right]^{2+}$ deviated from the model described in Sec. II, while $\left[\mathrm{Fe}\left(\mathrm{H}_{2} \mathrm{O}\right)_{6}\right]^{2+}$ provided excellent agreement. This behavior can be understood through further analyses of the nature of the ZFS as a function of $\Delta$, the energies of the ${ }^{5} \mathrm{E}_{\mathrm{g}}(1 \mathrm{a}, 1 \mathrm{~b})$ states in Fig. 1 . When $\Delta$ is $<\sim 500 \mathrm{~cm}^{-1}$, the axial ZFS is not well-described by Eq. (1). ${ }^{31}$ Rather, deviations from Eq. (1) can occur due to the presence of in-state orbital angular momentum deriving from the parent ${ }^{5} \mathrm{~T}_{2 \mathrm{~g}}$ state. More specifically, from Eq. (1), the ZFS value is predicted to exhibit an inverse dependence on $\Delta$ and, thus, approaches infinity as $\Delta$ approaches zero (i.e., for a ${ }^{5} \mathrm{~T}_{2 \mathrm{~g}}$ ground state, black dashed line in Fig. 4). This is not observed, however, upon use of a full spin Hamiltonian including SOC over all components of the ${ }^{5} \mathrm{~T}_{2 \mathrm{~g}}$ ground state. ${ }^{31}$ In this latter case, a downturn in the energy of the $\mathrm{D}$ value occurs when $\Delta$ is $<\sim 500 \mathrm{~cm}^{-1}$. Thus, from this comparison, potential deviations between the left-hand and right-hand sides of Eq. (3) are expected when $\Delta$ is $<\sim 500 \mathrm{~cm}^{-1}$. Indeed, the average values of the ${ }^{5} \mathrm{E}_{\mathrm{g}}(1 \mathrm{a}, 1 \mathrm{~b})$ excited states, $\Delta$, for the $\left[\mathrm{Fe}\left(\mathrm{H}_{2} \mathrm{O}\right)_{6}\right]^{2+}$ structures are $2095 \mathrm{~cm}^{-1}$ and $635 \mathrm{~cm}^{-1}$ for the neutron diffraction and optimized structures, respectively. The analogous values for the $\mathrm{x}$-ray idealized and optimized structures of $\left[\mathrm{Fe}\left(\mathrm{NH}_{3}\right)_{6}\right]^{2+}$ are $190 \mathrm{~cm}^{-1}$ and $180 \mathrm{~cm}^{-1}$, respectively, well below $500 \mathrm{~cm}^{-1}$. Thus, for the $\left[\mathrm{Fe}\left(\mathrm{NH}_{3}\right)_{6}\right]^{2+}$ structures, we ascribe the deviations of linear spin-phonon coupling terms estimated from either the left-hand or right-hand sides of Eq. (3) to the breakdown of Eq. (1) upon increase in the amount of in-state orbital angular momentum from the low energies of the ${ }^{5} \mathrm{E}_{\mathrm{g}}(1 \mathrm{a}, 1 \mathrm{~b})$ states ( $\Delta$ in Fig. 4 ).

To gain further insights into the direct calculation of linear spin-phonon coupling terms and to better understand the behavior of the CASSCF/NEVPT2 calculated axial ZFS as a function of the ${ }^{5} \mathrm{E}_{\mathrm{g}}(1 \mathrm{a}, 1 \mathrm{~b})$ states, $\Delta$, the totally symmetric $a_{1 g}$ mode can be used as a means to determine the functional form of $\mathrm{D}$ vs $\Delta$ (Fig. 4). The variation in $\mathrm{D}$ as a function of $\Delta$, as predicted by Eq. (1) $\left(\lambda=-100 \mathrm{~cm}^{-1}\right)$, is given in Fig. 4 (black dashed line). The CASSCF/NEVPT2 calculated correlations for $\left[\mathrm{Fe}\left(\mathrm{H}_{2} \mathrm{O}\right)_{6}\right]^{2+}$ and $\left[\mathrm{Fe}\left(\mathrm{NH}_{3}\right)_{6}\right]^{2+}$ are also given in Fig. 4 (colored squares and corresponding solid lines; see legend for color labeling). For comparison, we also provide the correlation generated using the BP86 optimized geometry of the high-spin form of $\left[\mathrm{Co}\left(\mathrm{NH}_{3}\right)_{6}\right]^{2+}$ (green square and line, Fig. 4) (the structure obtained here is consistent with the excited state distorted structure given by Solomon and co-workers ${ }^{56}$ ). From Eq. (1), it can 


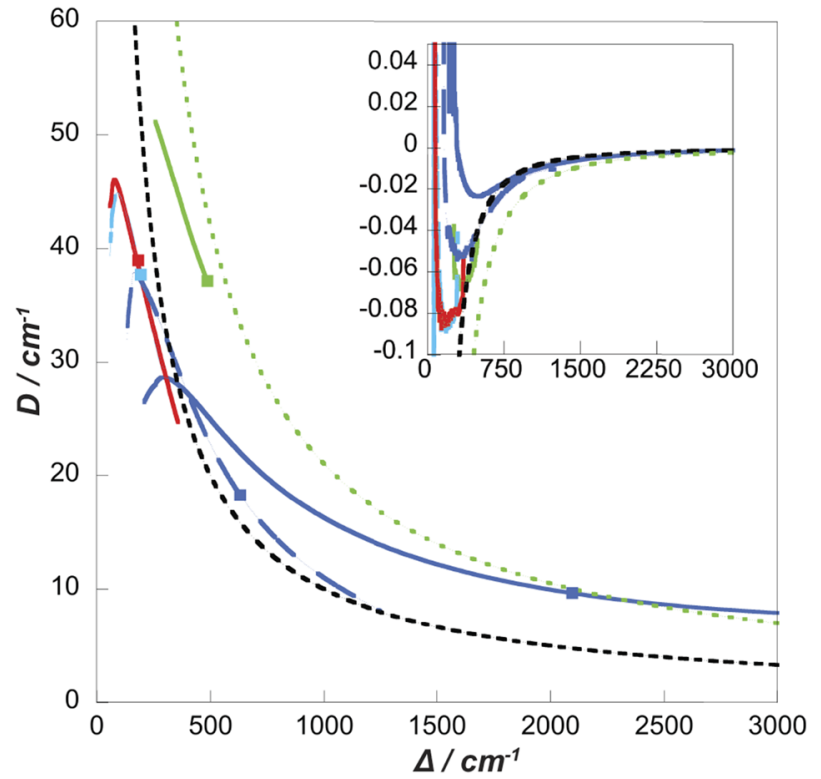

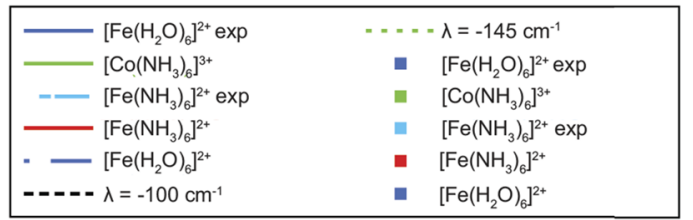

FIG. 4. Variations of $+D$ vs $\Delta$ [the energies of the ${ }^{5} E_{g}(1 a, 1 b)$ states given in Fig. 1] using Eq. (1) for different values of $\lambda\left(-100 \mathrm{~cm}^{-1}\right.$, dashed black, and $-145 \mathrm{~cm}^{-1}$, dashed green lines) compared to CASSCF/NEVPT2 computed values (color scheme given in the figure legend). (Inset) Magnitude of the first derivative of the $D$ vs $\Delta$. In the legend, exp represents data for the neutron diffraction and the x-ray crystallographically derived structures of $\left[\mathrm{Fe}\left(\mathrm{H}_{2} \mathrm{O}\right)_{6}\right]^{2+}$ and $\left[\mathrm{Fe}\left(\mathrm{NH}_{3}\right)_{6}\right]^{2+}$, respectively. As noted in the text, Eq. (1) predicts the value of the ZFS, D, to approach infinity as the value of the energies of the ${ }^{5} E_{g}(1 a, 1 b)$ states approach zero (i.e., for a ${ }^{5} \mathrm{~T}_{2 g}$ ground state). However, the use of a full spin Hamiltonian including SOC over all components of the ${ }^{5} \mathrm{~T}_{2 g}$ ground state ${ }^{31}$ corrects for the presence of in-state orbital angular momentum and leads to a downturn in the ZFS values as $\Delta$ drops below $\sim 500 \mathrm{~cm}^{-1}$. This is also observed here for the CASSCF/NEVPT2 calculations on several Fe(II) complexes. The colored squares correspond to the values of the ZFS and $\Delta$ at the equilibrium geometries, while the corresponding lines for a given structure (i.e., the variations of $+D$ vs $\Delta$ ) are obtained using the $a_{1 g}$ vibrational mode.

be seen that $\mathrm{D}$ increases due to the increased SOC constant of Co(III) $\left(580 \mathrm{~cm}^{-1}\right)$ relative to $\mathrm{Fe}(\mathrm{II})\left(400 \mathrm{~cm}^{-1}\right)$. Note that the shapes of the CASSCF/NEVPT2 calculated traces relative to those obtained using Eq. (1) (black dashed line) are similar to those obtained from the full spin Hamiltonian including SOC over all components of the ${ }^{5} \mathrm{~T}_{2 \mathrm{~g}}$ ground state. ${ }^{31}$ In particular, a downturn of the value of $\mathrm{D}$ is observed here. Furthermore, the first derivatives of $\mathrm{D}$ vs $\Delta$ are given in the inset of Fig. 4. Significant deviations between the first derivative of Eq. (1) and those calculated from the first derivative of the CASSCF/NEVPT2 calculations are observed at energies $<\sim 500 \mathrm{~cm}^{-1}$ (i.e., around energies where the value of D does not continue to increase but exhibits the downturn in energy, consistent with the full spin Hamiltonian description ${ }^{31}$ ).

In summary, deviations due to in-state orbital angular momentum can give rise to deviations between the linear spin-phonon coupling terms estimated by Eq. (1) and, thus, those from CASSCF/NEVPT2 calculations and the left-hand or right-hand sides of Eqs. (2) and (3).

\section{Extension to the ${ }^{1} \mathrm{~A}_{1 \mathrm{~g}} /{ }^{5} \mathrm{~T}_{2 \mathrm{~g}} \Delta \mathrm{S}=2$ ISC}

\section{ISC through the lens of ZFS}

A Tanabe-Sugano-like diagram generated with the $a_{1 g}$ mode for the $\left[\mathrm{Fe}\left(\mathrm{H}_{2} \mathrm{O}\right)_{6}\right]^{2+}$ neutron diffraction structure is given in Fig. 5(a). As expected, a clear change in ground state spin occurs along the $a_{1 g}$ breathing mode [e.g., the kink and red vertical line in Figs. 5(a) and 5(b)]. Given the CASSCF/NEVPT2 methodology can quantitatively follow the energetic evolution of the ZFS and lowlying $M_{S}$ sublevels of the ground state, we sought to explore and analyze the mechanism of ISC. To do so, the energies of the five $M_{S}$ sublevels of the ground state and the ${ }^{1} \mathrm{~A}_{1 \mathrm{~g}}$ excited state were followed with high resolution steps near the ISC [Fig. 5(b)]. Note that the lowest $M_{S}$ sublevel is set to zero energy in Figs. 5(a) and 5(b). An $a_{1 g}$ PES without normalizing to the ground state energy is also given in Fig. 6(a). From Fig. 5(b), the fifth highest energy excited state [originally the ${ }^{1} \mathrm{~A}_{1 \mathrm{~g}}$ excited state (black line)] approaches the $M_{S}= \pm 2$ and \pm 1 sublevels (green and purple lines) and then crosses the $M_{S}= \pm 2$ sublevels. However, ISC (as deduced from the lowest energy component of the CASSCF/NEVPT2 transition energies) does not occur until some point after the original ${ }^{1} \mathrm{~A}_{1 \mathrm{~g}}$ excited state continues to approach the $M_{S}= \pm 1$ sublevels, with ISC occurring at the vertical red line in Fig. 5(b). Furthermore, as the original ${ }^{1} \mathrm{~A}_{1 \mathrm{~g}}$ state approaches the orbitally nondegenerate ground state along the $a_{1 g}$ PES, it undergoes mixing with the lowest energy $M_{S}$ component. The amount of singlet character in the original ${ }^{1} \mathrm{~A}_{1 \mathrm{~g}}$ excited state is given by the black dashed line in Fig. 5(b), while the amount of singlet character mixed into the ground state is given by the brown dashed line. This increase in ${ }^{1} \mathrm{~A}_{1 \mathrm{~g}}$ excited state contribution to the lowest energy, ground state $M_{S}$ sublevel occurs with a concomitant decrease in the ${ }^{1} \mathrm{~A}_{1 \mathrm{~g}}$ contribution to the original ${ }^{1} \mathrm{~A}_{1 \mathrm{~g}}$ excited state. Note that the energy separation between the lowest energy $M_{S}$ sublevel and the original ${ }^{1} \mathrm{~A}_{1 \mathrm{~g}}$ state at ISC is $\sim 100 \mathrm{~cm}^{-1}$, which we tentatively describe as the electronic coupling term, $\mathrm{V}$ (not the rhombic ZFS V). This value is consistent with values considered previously for describing spin conversion processes in $\mathrm{Fe}(\mathrm{II})$ complexes. Finally, as the original ${ }^{1} \mathrm{~A}_{1 \mathrm{~g}}$ excited state crosses the $M_{S}= \pm 2$ sublevels, its singlet character is transferred to the third excited state (red solid and dashed lines).

As discussed further, while only the $a_{1 g}$ PES is followed here, other vibrational modes will likely play important roles in perturbing this picture and facilitate the overall mixings. For example, McCusker et al. ${ }^{59}$ and Purcell ${ }^{60}$ discuss the role of trigonal twisting modes. In principle, all the different mode contributions can be determined by evaluating the linear spin-phonon and excited state coupling terms along each vibrational mode.

As the ${ }^{1} \mathrm{~A}_{1 \mathrm{~g}}$ and ${ }^{5} \mathrm{~T}_{2 \mathrm{~g}}$ states differ by $\Delta \mathrm{S}=2$, no matrix elements connect them directly. However, nonzero matrix elements exist between both the ${ }^{1} \mathrm{~A}_{1 \mathrm{~g}}$ and ${ }^{5} \mathrm{~T}_{2 \mathrm{~g}}$ states with the ${ }^{3} \mathrm{~T}_{1 \mathrm{~g}}$ state. 

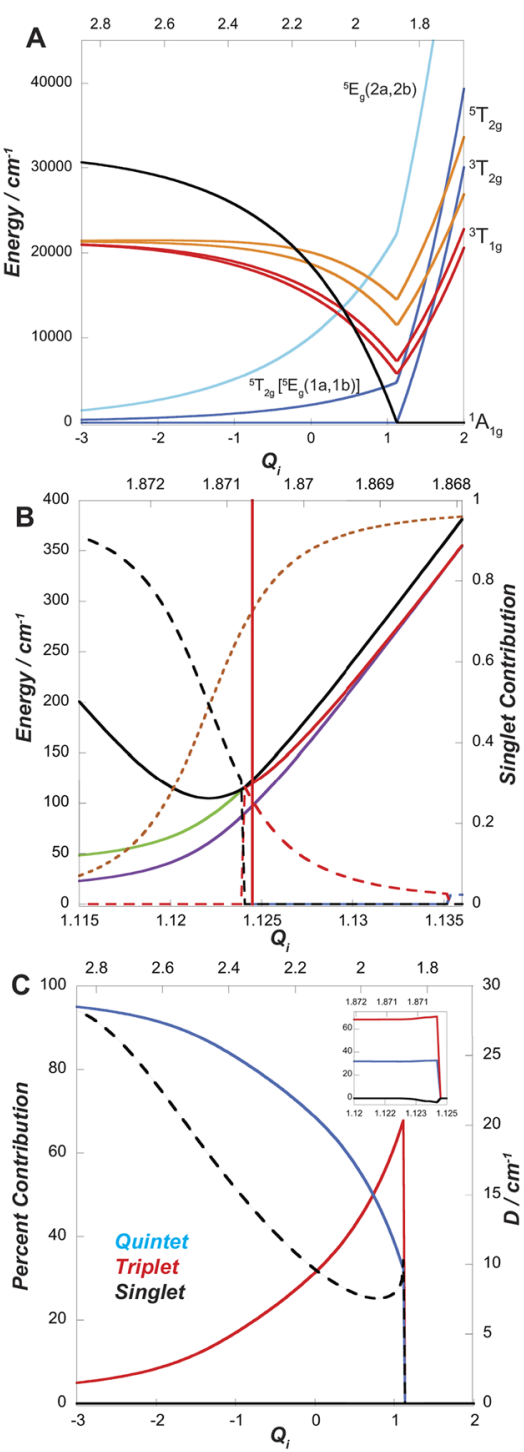

FIG. 5. Electronic structural changes along the $a_{1 g}$ mode of the neutron diffraction structure of $\left[\mathrm{Fe}\left(\mathrm{H}_{2} \mathrm{O}\right)_{6}\right]^{2+}$. (a) State energies obtained from CASSCF/NEVPT2 calculations, normalized to the ground state energy, exhibiting Tanabe-Sugano-like behavior. (b) Energies (solid lines) of the lowest five excited states, normalized to the ground state and accounting for ZFS, near the ISC point (vertical red line). The energy orderings (from low to high) are blue, purple, red, green, and black. The blue and red lines are degenerate with the purple and green lines, and thus only the purple and green lines are visible. Dashed lines show the contribution of the lowest energy singlet to each excited state. For example, the total singlet character in the fifth excited state (originally the ${ }^{1} A_{1 g}$ excited state) is given by the dashed black line, while the singlet contribution to the ground state is depicted by the brown dashed line. As the dominantly singlet excited state (solid black line) approaches the ground state quintet sublevels (blue/purple and red/green sublevels are degenerate, respectively), the singlet character is transferred to the third excited state (red line, originally beneath the green line). Thus, the dashed red line depicts the residual singlet excited state character in that specific excited state. (c) Variations in the percent contributions to $+D$ along the $a_{1 g}$ mode (solid lines) vs the value of ZFS, D (dashed black line). The break occurs at the ISC point. (Inset) Zoom in of the region near ISC where the singlet states exhibit a small negative contribution to the ZFS, D (top x-axis units throughout: average Fe-O bond length, $\AA$ ).
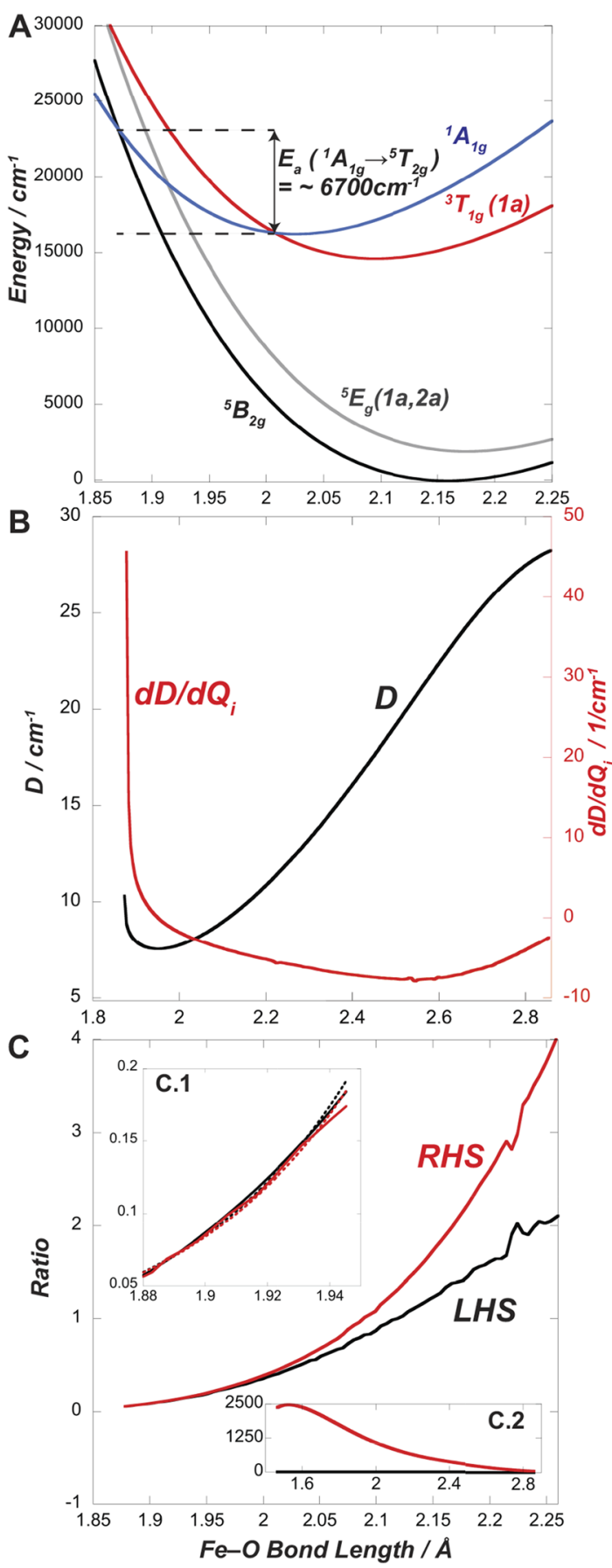

FIG. 6. Variations of ligand field and spin Hamiltonian parameters along the $a_{19}$ mode of the $\left[\mathrm{Fe}\left(\mathrm{H}_{2} \mathrm{O}\right)_{6}\right]^{2+}$ neutron diffraction structure. (a) Ground and excited state PESs relevant for the $\triangle S=2$ ISC. (b) Variation of $D$ (black line) and its first derivative (red line). (c) Ratio of the linear spin-phonon coupling terms computed using the left-hand side (LHS) (black line) and right-hand side (RHS) (red line) of Eq. (6). (Inset, C.1) Zoom in of the ratios near the ISC point, including exponential fits (dotted lines) $\left(R^{2}=>0.99\right)$. [Inset, (c.2)] Energy splitting in $\mathrm{cm}^{-1}$ of the orbitally degenerate ${ }^{3} \mathrm{~T}_{1 \mathrm{~g}}$ excited state (in $\mathrm{O}_{h}$ ). The lowest energy component (black line) is normalized to zero energy. 
Because of this, the triplet is thought to mediate the ISC. ${ }^{57}$ The ${ }^{1} \mathrm{~A}_{1 \mathrm{~g}}$ mixings described above can be probed by following the same PES without triplet states (e.g., 5 quintets and 15 singlets). Doing so eliminates the mixing of the ${ }^{1} \mathrm{~A}_{1 \mathrm{~g}}$ excited into the ground state $M_{S}$ sublevel (Fig. S3).

In addition to understand the ${ }^{1} \mathrm{~A}_{1 \mathrm{~g}}$ state contributions over the ground state $M_{S}$ sublevels, the individual contributions to the overall ZFS from quintet, triplet, and singlet states along the $a_{1 g}$ PES can be evaluated [Fig. 5(c)]. As mentioned above, the quintet states are the major contributor to $+\mathrm{D}$ at the equilibrium geometry. However, as the structure evolves along the $a_{1 g} \mathrm{PES}$, the percent contribution to $\mathrm{D}$ from the triplets increases dramatically. Indeed, just before the ${ }^{1} \mathrm{~A}_{1 \mathrm{~g}}$ state crosses the $M_{S}= \pm 2$ sublevels $(\sim 1.871 \AA$ Fe-O bond distance), the major contribution to the ZFS is from the triplet states $(\sim 70 \%)$, with a significantly smaller contribution from the quintets $(30 \%)$ [Fig. 5(c)]. Also, after the original ${ }^{1} \mathrm{~A}_{1 \mathrm{~g}}$ state crosses the $M_{S}= \pm 2$ sublevels, just before ISC, even the singlet states contribute a small amount to D [Fig. 5(c), inset]. Thus, in this case, while ISC is not mediated by the triplet excited state through a direct population, the overall contributions to the ZFS reflect a dominant triplet component $\left({ }^{3} \mathrm{~T}_{1 \mathrm{~g}}\right)$ from excited state SOC. This increased triplet component further allows for the ${ }^{1} \mathrm{~A}_{1 \mathrm{~g}}$ mixing into the ground state $M_{S}$ sublevels, facilitating ISC.

In summary, the magnitude and nature of the ZFS evolves significantly along the $a_{1 g}$ PES. This necessarily requires concomitant changes in the magnitudes and natures of the linear spin-phonon coupling terms. These observations stem directly from the dynamic nature of the linear excited state coupling terms over the potential energy landscape. This method, employing CASSCF/NEVPT2 calculations and analyses, represents a powerful means to dissect the mechanisms of SCO and ISCs in transition metal dynamics and photophysics.

\section{A spin-phonon coupling-based mechanism of ISC}

As discussed above, the $M_{S}$ sublevels of the orbitally nondegenerate component of the $\mathrm{S}=2$ ground state undergo mixing with the ${ }^{1} \mathrm{~A}_{1 \mathrm{~g}}$ excited state along the $a_{1 g}$ vibrational coordinate. This mixing is facilitated by the ${ }^{3} \mathrm{~T}_{1 \mathrm{~g}}$ excited state. Thus, here, using the neutron diffraction structure of $\left[\mathrm{Fe}\left(\mathrm{H}_{2} \mathrm{O}\right)_{6}\right]^{2+}$, we further consider the role of spin-phonon coupling in facilitating ISC. We have approached this with the following steps: (1) the energies of the ${ }^{1} \mathrm{~A}_{1 \mathrm{~g}},{ }^{5} \mathrm{E}_{\mathrm{g}}(1 \mathrm{a}, 1 \mathrm{~b})$, and the ${ }^{3} \mathrm{~T}_{1 \mathrm{~g}}$ states were calculated along the $a_{1 g}$ PES [Fig. 6(a)]. Note that this provides an activation energy, $E_{a}$, of $\sim 6700 \mathrm{~cm}^{-1}$ for ${ }^{1} \mathrm{~A}_{1 \mathrm{~g}} \rightarrow{ }^{5} \mathrm{~T}_{2 \mathrm{~g}}$ ISC. This value will be used for further analyses presented below. (2) The ZFS and its derivative were calculated along the $a_{1 g}$ PES [Fig. 6(b)]. While the ZFS ranges from $\sim 7 \mathrm{~cm}^{-1}$ to $15 \mathrm{~cm}^{-1}$ along this PES, there is a dramatic increase in ZFS and the corresponding linear spin-phonon coupling term as the structure approaches the ISC point. Given the PES is along the $a_{1 g}$ mode, we can convert the distortion to an $\AA^{-1}$ scale. (3) Using this, linear excited state coupling terms for the ${ }^{5} \mathrm{E}_{\mathrm{g}}(1 \mathrm{a}, 1 \mathrm{~b})$ and ${ }^{3} \mathrm{~T}_{1 \mathrm{~g}}$ states along the $a_{1 g}$ PES can be obtained. Note that the ${ }^{3} \mathrm{~T}_{1 \mathrm{~g}}$ state splits into a lower energy nondegenerate $\left[{ }^{3} \mathrm{~T}_{1 g}(1 \mathrm{a})\right]$ and higher energy degenerate set of states $\left[{ }^{3} \mathrm{~T}_{1 \mathrm{~g}}(1 \mathrm{~b}, 1 \mathrm{c})\right]$. Near the ISC, it is ${ }^{3} \mathrm{~T}_{1 \mathrm{~g}}(1 \mathrm{a})$ that largely contributes to $+\mathrm{D}$, while the other ${ }^{3} \mathrm{~T}_{1 \mathrm{~g}}(1 \mathrm{~b}, 1 \mathrm{c})$ states contribute negatively. (4) Linear spin-phonon coupling terms were then calculated for the ${ }^{5} \mathrm{E}_{\mathrm{g}}(1 \mathrm{a})$ and ${ }^{3} \mathrm{~T}_{1 \mathrm{~g}}(1 \mathrm{a})$ states along the $a_{1 g}$ PES. (5) The ratios of the linear spin-phonon coupling terms between the ${ }^{5} \mathrm{E}_{\mathrm{g}}(1 \mathrm{a})$ and ${ }^{3} \mathrm{~T}_{1 \mathrm{~g}}$ (1a) states were then evaluated along the $a_{1 g}$ PES [Fig. 6(c), red line] [i.e., using the right-hand side of Eq. (6)]. Interestingly, this ratio of linear spin-phonon coupling terms $\left[{ }^{5} \mathrm{E}_{\mathrm{g}}(1 \mathrm{a}) /{ }^{3} \mathrm{~T}_{1 \mathrm{~g}}(1 \mathrm{a})\right]$ varies in a pseudo-exponential fashion over the $a_{1 g} \mathrm{PES}$, with a significant decrease in the ratio as the structure approaches the ISC point due to the increased contribution from the ${ }^{3} \mathrm{~T}_{1 \mathrm{~g}}(1 \mathrm{a})$ state and decreased contribution from the ${ }^{5} \mathrm{E}_{\mathrm{g}}(1 \mathrm{a}, 1 \mathrm{~b})$ states [also shown in Fig. 5(c)]. (6) The ratios of the linear spin-phonon coupling terms were also determined independently using the ZFS values calculated using the CASSCF/NEVPT2 approach [i.e., the left-hand side of Eq. (6)]. Thus, the ratios obtained using both sides of Eq. (6) can be compared directly [Fig. 6(c), red vs black lines, respectively; a scaling factor, $n$, of 0.1 was employed here for the right-hand side of Eq. (6)].

Over the majority of the $a_{1 g}$ scan, the agreement between the two sides of Eq. (6) is poor [Fig. 6(c), red vs black line]. However, the agreement improves dramatically nearer the region of ISC. As outlined in Sec. II C 3 for $\left[\mathrm{Fe}\left(\mathrm{H}_{2} \mathrm{O}\right)_{6}\right]^{2+}$ vs $\left[\mathrm{Fe}\left(\mathrm{NH}_{3}\right)_{6}\right]^{2+}$, the disagreement with Eq. (2) for these structures derived from the presence of in-state orbital angular momentum. Since the ${ }^{3} \mathrm{~T}_{1 \mathrm{~g}}$ state is threefold orbitally degenerate, it will contain in-state orbital angular momentum, the magnitude of which should be larger for a free ion vs that in a complex. Accordingly, the three components of the ${ }^{3} \mathrm{~T}_{1 \mathrm{~g}}$ excited state are split in energy along the $a_{1 g}$ PES and approaching the ISC point; this splitting quenches the in-state orbital angular momentum [Fig. 6(c), inset (c.2)]. Thus, better agreement between the left-hand and right-hand sides of Eq. (6) is obtained when instate orbital angular momentum of the ${ }^{3} \mathrm{~T}_{1 \mathrm{~g}}$ excited state is quenched by the structural distortion, which corresponds to structures that are closer to the ISC point.

Thus, overall, while the spin-phonon coupling terms corresponding to the magnetization dynamics of the ${ }^{5} \mathrm{~B}_{2 \mathrm{~g}}$ ground state along the $a_{1 g}$ mode facilitating ISC are dominantly associated with the ${ }^{5} \mathrm{E}_{\mathrm{g}}(1 \mathrm{a}, 1 \mathrm{~b})$ states near the ground state equilibrium geometry, the nature and the state-specific contributions to the ground state spin-phonon coupling terms evolve significantly as the structure approaches the ISC point and grow to become dominated by contributions from the ${ }^{3} \mathrm{~T}_{1 \mathrm{~g}}(1 \mathrm{a})$ state. For the structure considered here, the in-state orbital angular momentum of the threefold orbitally degenerate ${ }^{3} \mathrm{~T}_{1 \mathrm{~g}}$ state is quenched along the $a_{1 g}$ mode, giving rise to better agreement between the left-hand and right-hand sides of Eq. (6). This may not be the case for other structures or other vibrational modes, however, and suggests that, in some cases, instate orbital angular momentum can contribute to the magnitude of spin-phonon coupling terms and, thus, the resulting magnetization dynamics. Equation (6), therefore, appears to encompass the important vibrationally driven SOC dynamics, which are ultimately themselves drivers of the ISC and are directly reflected in the ZFS dynamics along specific PESs.

While not the main focus of this section, given the variation in the ratios of the ${ }^{5} \mathrm{E}_{\mathrm{g}}(1 \mathrm{a}, 1 \mathrm{~b})$ and ${ }^{3} \mathrm{~T}_{1 \mathrm{~g}}(1 \mathrm{a})$ linear spin-phonon coupling terms appear to change in a pseudo-exponential fashion, we fit the change in the ratio near the ISC for the results from the lefthand and right-hand sides of Eq. (6) [Fig. 6(c), inset (c.1)] to a single exponential function. Note that the data in Fig. 6(c) have been converted to $\AA^{-1}$. For the right-hand side, the fit provides an exponent 
of 17.492 , while a value of 17.150 is obtained from the fit of the lefthand side. While the ratio of linear spin-phonon coupling terms is unitless, it is interesting to calculate an "apparent activation energy, $\mathrm{E}_{\mathrm{a}}$," assuming an Arrhenius model. Converting the exponent to an energy at $295 \mathrm{~K}$ and multiplying by the approximate $\mathrm{Fe}-\mathrm{O}$ bond distance near the ISC point ( $\sim 1.878 \AA$ taken here) provides energies of $\sim 6605 \mathrm{~cm}^{-1}$ and $\sim 6735 \mathrm{~cm}^{-1}$ for the left-hand and right-hand sides of Eq. (6), respectively. Interestingly, the " $E_{a}$ " determined in this way is very similar to that of $\sim 6700 \mathrm{~cm}^{-1}$ determined directly in Fig. 6(a) for the ${ }^{1} \mathrm{~A}_{1 \mathrm{~g}} \rightarrow{ }^{5} \mathrm{~T}_{2 \mathrm{~g}}$ interconversion. Note that the " $\mathrm{E}_{\mathrm{a}}$ " determined from the ratio of intramolecular spin-phonon coupling terms does not feature the ${ }^{1} \mathrm{~A}_{1 \mathrm{~g}}$ energy directly; rather, the model only takes into account the dynamic ZFS contributions from the ${ }^{5} \mathrm{E}_{\mathrm{g}}(1 \mathrm{a}, 1 \mathrm{~b})$ and ${ }^{3} \mathrm{~T}_{1 \mathrm{~g}}$ states near the ISC. Note that we do not claim this to be a general means of determining an $\mathrm{E}_{\mathrm{a}}$ (hence, the quotations around apparent activation energy), but it is certainly interesting to note this observation in passing here within the broader context of the model. It will be of interest to consider this further and whether the model can accurately describe apparent activation energies obtained from variable temperature rates of Fe(II) ISCs measured by transient absorption spectroscopy.

In summary, the ratios of intramolecular spin-phonon coupling terms deriving from different excited states reflect dynamic ZFS contributions along vibrational PESs and form the basis for a spin-phonon coupling-based model of ISC. The ratios determined using the left-hand or right-hand sides of Eq. (6) are also sensitive to the amount in-state orbital angular momentum in the specific excited states that govern the spin-phonon coupling terms. These dynamic ZFSs drive ISC in transition metal complexes. Furthermore, while the analyses are only presented here for a single vibrational mode, others will undoubtedly be of interest, especially upon symmetry lowering. Future studies will be directed at extending the model to other Fe(II) complexes, multidimensional PESs, and transition metal ISC dynamics in general.

\section{DISCUSSION}

The phenomenon of spin-phonon coupling manifests in a wide array of important aspects of transition metal dynamics. Here, we have developed a combined ligand field theory model and CASSCF/NEVPT2 computational approach to identify and describe linear spin-phonon coupling terms for $\mathrm{S}=2 \mathrm{Fe}(\mathrm{II})$ complexes with $+\mathrm{D}$. This description allows for the determination of the ligand field excited state origin of ground state linear spin-phonon coupling terms, which are quite sensitive to the geometric and electronic structure of the complex. As such, the overall coupling terms for different structures can vary significantly for specific normal modes [e.g., $a_{1 g}, e_{g}(\theta)$, and $e_{g}(\varepsilon)$ modes] and activate modes in a complex that were inactive in others. We have further shown that the ligand field theory model described in Sec. II can break down when significant in-state orbital angular momentum is present in the ground state $\left\{\right.$ e.g., for very low energy excited states $\left[{ }^{5} \mathrm{E}_{\mathrm{g}}(1 \mathrm{a}, 1 \mathrm{~b})\right.$ in this study] $\}$ or when couplings are mediated by orbital triplet excited states (e.g., ${ }^{3} \mathrm{~T}_{1 \mathrm{~g}}$ ). Nonetheless, direct comparisons with multireference $a b$ initio calculations allow for significant insights into the nature of spin-phonon coupling, and the model is directly translatable to other transition metal complexes.

\section{A. Covalency contributions}

While complexes considered here are in an ionic regime, it will be of interest to extend the models to complexes that exhibit higher degrees of covalency. For example, anisotropic covalency has been shown to contribute significantly to the ZFSs in transition metal complexes (e.g., $\left[\mathrm{FeCl}_{4}\right]^{-}$and $\left.\left[\mathrm{Co}(\mathrm{SPh})_{4}\right]^{2-}\right) \cdot{ }^{26,27,35}$

\section{B. Experimental considerations}

Additional questions remain as to how spin-phonon coupling terms can be qualitatively or quantitatively evaluated experimentally. A recent study utilizing magnetic field dependent Raman and far-IR studies has observed avoided crossings, which have been utilized to evaluate and quantify spin-phonon coupling. ${ }^{6,7}$ However, it should be noted that the linear spin-phonon coupling terms discussed here are fundamentally different from the couplings discussed in these references, as they deal with the case where a vibrational mode and an $M_{S}$ sublevel are nearly degenerate and undergo direct interactions. That said given they span 4D in energy, these zero-field split $M_{S}$ sublevels could approach $100 \mathrm{~cm}^{-1}$, which would put them near coherent vibrational energies that are directly involved in ISC (vide infra). ${ }^{17}$ Furthermore, there have been reports of magnetic field dependent rates of ISC in $\mathrm{Ni}$ (II) complexes. ${ }^{63,64}$ Indeed, the model used to interpret these magnetic field dependent ISC rates invoked large values of excited state ZFS $\left(\sim+24 \mathrm{~cm}^{-1}\right){ }^{63}$ Additionally, different molecular symmetries also gave rise to different magnetic field dependencies on ISC rates, suggesting that different values of ZFSs may manifest in these field dependent rates. ${ }^{64}$ Additionally, significant magnetic field effects have been observed on the rates of cooperative SCO for $\left[\mathrm{Fe}(2-\mathrm{pic})_{3}\right] \mathrm{Cl}_{2} \cdot \mathrm{EtOH}$ (2-pic $=2$-picolylamine). ${ }^{65}$ While the authors observed little effect on the transition temperature for SCO $(0.2 \mathrm{~K})$, increased magnetic fields dramatically accelerated the rate of formation of the $S=2$ state through the LIESST effect $(710 \%$ at $7 \mathrm{~T}$ relative to $0.5 \mathrm{~T})$. Also, at $10 \mathrm{~K}$, an increased magnetic field dramatically decreased the rate of relaxation to the $S=0$ ground state. These combined observations of magnetic field effects on rates of ISC/SCO, together with the observations described in this work, strongly motivate future combined steady state and ultrafast magnetic resonance and optical experiments to measure and quantify variable temperature variable field (VTVH) ISC/SCO kinetics.

\section{Implications for ISC/SCO and photophysics}

An interesting observation here is that the $a_{1 g}$ breathing mode, which has been implicated in ISC/SCO dynamics, ${ }^{14,16-18}$ exhibits appreciable linear spin-phonon and excited state coupling terms, demonstrating that the methodology can successfully identify the molecular motions coupled to spin conversion processes. Following the $a_{1 g}$ modes in $\mathrm{Fe}$ (II) complexes considered here leads directly to ISC and, thus, provides an opportunity to study the spinphonon coupling-based mechanism of spin conversion as predicted by CASSCF/NEVPT2 calculations. Indeed, in our preliminary evaluations of spin-phonon coupling terms in $\left[\mathrm{Fe}(\mathrm{bpy})_{3}\right]^{2+}$, the $a_{1 g}$ breathing mode calculated at $\sim 120 \mathrm{~cm}^{-1}$, which has been identified as the key mode for the ultrafast formation of the ${ }^{5} \mathrm{~T}_{2 \mathrm{~g}}$ state, ${ }^{17}$ is one of the several modes that exhibit appreciable linear spin-phonon coupling terms. These results, as well as a comparison to other 
photo-active $\mathrm{Fe}(\mathrm{II})$ complexes, will be presented in a forthcoming study.

Overall, the model presented here suggests that dynamic ZFSs, which are directly reflected in spin-phonon coupling terms, play a key role and allow for the determination of individual excited state contributions to the ZFS at different positions along a PES. For example, while the ${ }^{5} \mathrm{E}_{\mathrm{g}}$ states contribute the majority of the ZFS at the equilibrium geometry of $\left[\mathrm{Fe}\left(\mathrm{H}_{2} \mathrm{O}\right)_{6}\right]^{2+}$, at the ISC, the spinforbidden ${ }^{3} \mathrm{~T}_{1 \mathrm{~g}}$ state (and to a small extent the singlets) is the dominant contributor. Concurrently, the ${ }^{3} \mathrm{~T}_{1 \mathrm{~g}}$ contribution to the ZFS is a direct reflection of its further role in turning on the ${ }^{1} \mathrm{~A}_{1 \mathrm{~g}}$ state mixing with the lowest energy $M_{S}$ sublevel (Fig. 5), consistent with the previously described mechanism of SCO.

The role of the ${ }^{3} \mathrm{~T}_{1 \mathrm{~g}}$ state in the ISC process has been studied in great detail using ultrafast spectroscopies. ${ }^{69-71}$ While the triplet state has been identified as a fleeting intermediate during the initial formation of the ${ }^{5} \mathrm{~T}_{2 \mathrm{~g}}$ state in $\mathrm{Fe}(\mathrm{II})$ polypyridyl compounds, ${ }^{69,70}$ we have mainly focused on understanding its role in the direct ${ }^{1} \mathrm{~A}_{1 \mathrm{~g}} /{ }^{5} \mathrm{~T}_{1 \mathrm{~g}}$ $\Delta S=2$ spin conversion, which typically governs the overall rate of spin conversion. ${ }^{61,72}$ An important finding here is the demonstration that the spin conversion process can be described by quantitatively evaluating the relative intramolecular linear spin-phonon coupling terms for the ${ }^{5} \mathrm{E}_{\mathrm{g}}(1 \mathrm{a}, 1 \mathrm{~b})$ and ${ }^{3} \mathrm{~T}_{1 \mathrm{~g}}$ states. A future study will focus on evaluating these ratios across $\mathrm{Fe}$ (II) complexes where rates and apparent activation energies have been measured using temperature dependent transient absorption spectroscopies. ${ }^{58,59}$ These general results can be further extended to the study of spin conversion processes in any $S>\frac{1}{2}$ transition metal complex.

\section{SUMMARY}

In summary, we have provided a means to evaluate linear spinphonon coupling terms in $\mathrm{S}=2 \mathrm{Fe}(\mathrm{II})$ complexes, which is useful for identifying modes that are involved in driving $\Delta S=2$ ISCs in transition metal complexes. Linear spin-phonon coupling terms originate from the presence of appreciable linear excited state coupling terms, are sensitive to the initial geometric and electronic structure of the transition metal complex, and will strongly influence ISC dynamics. It is further demonstrated that the CASSCF/NEVPT2 methodology provides a convenient means to describe the mechanism of spin conversion processes and highlights the role of dynamic ZFSs, which manifest in the relative ratios of intramolecular linear excited state coupling terms [Fig. 6(c) and Sec. II D 2] and dominate the mechanism of ISC. Thus, we envision insights from linear spin-phonon and excited state coupling terms can guide the synthetic modification of transition metal complexes for photomagnetic applications, efficient solar energy conversion, and photoredox catalysis. Finally, this study further connects concepts in magnetization dynamics across fields such as photophysics and quantum coherence.

\section{SUPPLEMENTARY MATERIAL}

See the supplementary material for computational methods, benchmark CASSCF/NEVPT2 calculations, supporting tables and figures, including DFT calculated vibrational modes and fits for linear spin-phonon and excited state coupling terms, Cartesian coordinates of all geometries, and representative ORCA input files.

\section{ACKNOWLEDGMENTS}

The authors acknowledge Dr. Jay Winkler for helpful discussions and Dr. Martin Srnec for assistance with initial CASSCF/NEVPT2 calculations. A.T.B. acknowledges funding through a National Science Foundation Graduate Research Fellowship (NSF Grant No. DGE-1745301). P.T.K. acknowledges funding through the Caltech Summer Undergraduate Research Fellowship (SURF) program. Financial support from Caltech and the Dow Next Generation Educator Fund is gratefully acknowledged.

\section{DATA AVAILABILITY}

The data that support the findings of this study are available from the corresponding author upon reasonable request.

\section{REFERENCES}

${ }^{1}$ L. Escalera-Moreno, J. J. Baldoví, A. Gaita-Ariño, and E. Coronado, Chem. Sci. 9, 3265 (2018).

${ }^{2}$ L. Escalera-Moreno, N. Suaud, A. Gaita-Ariño, and E. Coronado, J. Phys. Chem. Lett. 8, 1695 (2017).

${ }^{3}$ A. Albino, S. Benci, L. Tesi, M. Atzori, R. Torre, S. Sanvito, R. Sessoli, and A. Lunghi, Inorg. Chem. 58, 10260 (2019).

${ }^{4}$ A. Lunghi, F. Totti, R. Sessoli, and S. Sanvito, Nat. Commun. 8, 14620 (2017).

${ }^{5}$ A. Lunghi, F. Totti, S. Sanvito, and R. Sessoli, Chem. Sci. 8, 6051 (2017).

${ }^{6}$ D. H. Moseley, S. E. Stavretis, K. Thirunavukkuarasu, M. Ozerov, Y. Cheng, L. L. Daemen, J. Ludwig, Z. Lu, D. Smirnov, C. M. Brown, A. Pandey, A. J. RamirezCuesta, A. C. Lamb, M. Atanasov, E. Bill, F. Neese, and Z.-L. Xue, Nat. Commun. 9, 2572 (2018).

${ }^{7}$ M. Atanasov and F. Neese, J. Phys.: Conf. Ser. 1148, 012006 (2018).

${ }^{8}$ R. Mirzoyan and R. Hadt, Phys. Chem. Chem. Phys. "The dynamic ligand field of a molecular qubit: decoherence through spin-phonon coupling," (published online, 2020).

${ }^{9}$ B. Gu and I. Franco, J. Phys. Chem. Lett. 9, 773 (2018).

${ }^{10}$ A. J. Fielding, S. Fox, G. L. Millhauser, M. Chattopadhyay, P. M. H. Kroneck, G. Fritz, G. R. Eaton, and S. S. Eaton, J. Magn. Reson. 179, 92 (2006).

${ }^{11}$ R. Orbach, Proc. Phys. Soc. 77, 821 (1961).

${ }^{12}$ K. N. Shrivastava, Phys. Status Solidi B 117, 437 (1983).

${ }^{13}$ J. H. Van Vleck, Phys. Rev. 57, 426 (1940).

${ }^{14}$ C. Bressler, C. Milne, V.-T. Pham, A. ElNahhas, R. M. van der Veen, W. Gawelda, S. Johnson, P. Beaud, D. Grolimund, M. Kaiser, C. N. Borca, G. Ingold, R. Abela, and M. Chergui, Science 323, 489 (2009).

${ }^{15}$ M. Chergui and E. Collet, Chem. Rev. 117, 11025 (2017).

${ }^{16}$ M. Cammarata, R. Bertoni, M. Lorenc, H. Cailleau, S. Di Matteo, C. Mauriac, S. F. Matar, H. Lemke, M. Chollet, S. Ravy, C. Laulhé, J.-F. Létard, and E. Collet, Phys. Rev. Lett. 113, 227402 (2014).

${ }^{17}$ H. T. Lemke, K. S. Kjær, R. Hartsock, T. B. van Driel, M. Chollet, J. M. Glownia, S. Song, D. Zhu, E. Pace, S. F. Matar, M. M. Nielsen, M. Benfatto, K. J. Gaffney, E. Collet, and M. Cammarata, Nat. Commun. 8, 15342 (2017).

${ }^{18}$ A. Marino, M. Cammarata, S. F. Matar, J.-F. Létard, G. Chastanet, M. Chollet, J. M. Glownia, H. T. Lemke, and E. Collet, Struct. Dyn. 3, 023605 (2015).

${ }^{19}$ K. Kunnus, M. Vacher, T. C. B. Harlang, K. S. Kjær, K. Haldrup, E. Biasin, T. B. van Driel, M. Pápai, P. Chabera, Y. Liu, H. Tatsuno, C. Timm, E. Källman, M. Delcey, R. W. Hartsock, M. E. Reinhard, S. Koroidov, M. G. Laursen, F. B. Hansen, P. Vester, M. Christensen, L. Sandberg, Z. Németh, D. S. Szemes, É. Bajnóczi, R. Alonso-Mori, J. M. Glownia, S. Nelson, M. Sikorski, D. Sokaras, H. T. Lemke, S. E. Canton, K. B. Møller, M. M. Nielsen, G. Vankó, K. Wärnmark, V. Sundström, P. Persson, M. Lundberg, J. Uhlig, and K. J. Gaffney, Nat. Commun. 11, 634 (2020).

${ }^{20}$ K. S. Kjær, T. B. V. Driel, T. C. B. Harlang, K. Kunnus, E. Biasin, K. Ledbetter, R. W. Hartsock, M. E. Reinhard, S. Koroidov, L. Li, M. G. Laursen, F. B. Hansen, P. Vester, M. Christensen, K. Haldrup, M. M. Nielsen, A. O. Dohn, M. I. Pápai, 
K. B. Møller, P. Chabera, Y. Liu, H. Tatsuno, C. Timm, M. Jarenmark, J. Uhlig, V. Sundstöm, K. Wärnmark, P. Persson, Z. Németh, D. S. Szemes, É. Bajnóczi, G. Vankó, R. Alonso-Mori, J. M. Glownia, S. Nelson, M. Sikorski, D. Sokaras, S. E. Canton, H. T. Lemke, and K. J. Gaffney, Chem. Sci. 10, 5749 (2019).

${ }^{21}$ H. Tatsuno, K. S. Kjær, K. Kunnus, T. C. B. Harlang, C. Timm, M. Guo, P. Chàbera, L. A. Fredin, R. W. Hartsock, M. E. Reinhard, S. Koroidov, L. Li, A. A. Cordones, O. Gordivska, O. Prakash, Y. Liu, M. G. Laursen, E. Biasin, F. B. Hansen, P. Vester, M. Christensen, K. Haldrup, Z. Németh, D. S. Szemes, É. Bajnóczi, G. Vankó, T. B. Van Driel, R. Alonso-Mori, J. M. Glownia, S. Nelson, M. Sikorski, H. T. Lemke, D. Sokaras, S. E. Canton, A. O. Dohn, K. B. Møller, M. M. Nielsen, K. J. Gaffney, K. Wärnmark, V. Sundström, P. Persson, and J. Uhlig, Angew. Chem., Int. Ed. 59, 364 (2020).

${ }^{22}$ D. M. Arias-Rotondo and J. K. McCusker, Chem. Soc. Rev. 45, 5803 (2016).

${ }^{23}$ J. D. Braun, I. B. Lozada, C. Kolodziej, C. Burda, K. M. E. Newman, J. van Lierop, R. L. Davis, and D. E. Herbert, Nat. Chem. 11, 1144 (2019).

${ }^{24}$ K. S. Kjær, N. Kaul, O. Prakash, P. Chábera, N. W. Rosemann, A. Honarfar, O. Gordivska, L. A. Fredin, K.-E. Bergquist, L. Häggström, T. Ericsson, L. Lindh, A. Yartsev, S. Styring, P. Huang, J. Uhlig, J. Bendix, D. Strand, V. Sundström, P. Persson, R. Lomoth, and K. Wärnmark, Science 363, 249 (2019).

${ }^{25}$ J. K. McCusker, Science 363, 484 (2019).

${ }^{26}$ J. C. Deaton, M. S. Gebhard, S. A. Koch, M. Millar, and E. I. Solomon, J. Am. Chem. Soc. 110, 6241 (1988).

${ }^{27}$ F. Neese and E. I. Solomon, Inorg. Chem. 37, 6568 (1998).

${ }^{28}$ R. Boča, Coord. Chem. Rev. 248, 757 (2004).

${ }^{29}$ A. Lunghi, arXiv:1912.04545 [Cond-Mat Physicsquant-Ph] (2019).

${ }^{30}$ M. S. Fataftah, M. D. Krzyaniak, B. Vlaisavljevich, M. R. Wasielewski, J. M. Zadrozny, and D. E. Freedman, Chem. Sci. 10, 6707 (2019).

${ }^{31}$ E. I. Solomon, E. G. Pavel, K. E. Loeb, and C. Campochiaro, Coord. Chem. Rev. 144, 369 (1995).

${ }^{32}$ C. Corliss and J. Sugar, J. Phys. Chem. Ref. Data 11, 135 (1982).

${ }^{33}$ V. P. Gnezdilov, V. V. Eremenko, A. V. Peschanskii, and V. I. Fomin, Fiz. Nizk. Tem. 17, 253 (1953).

${ }^{34}$ E. I. Solomon, Comments Inorg. Chem. 3, 227 (1984).

${ }^{35}$ E. A. Suturina, D. Maganas, E. Bill, M. Atanasov, and F. Neese, Inorg. Chem. 54, 9948 (2015).

${ }^{36}$ M. Atanasov, D. Aravena, E. Suturina, E. Bill, D. Maganas, and F. Neese, Coord. Chem. Rev. 289-290, 177 (2015).

${ }^{37}$ M. Atanasov, D. Ganyushin, D. A. Pantazis, K. Sivalingam, and F. Neese, Inorg. Chem. 50, 7460 (2011).

${ }^{38} \mathrm{C}$. Sousa and C. de Graaf, Spin States in Biochemistry and Inorganic Chemistry (Wiley-Blackwell, 2015), pp. 35-57.

${ }^{39}$ C. Sousa, A. Domingo, and C. de Graaf, Chem. - Eur. J. 24, 5146 (2018).

${ }^{40}$ C. Sousa, C. de Graaf, A. Rudavskyi, R. Broer, J. Tatchen, M. Etinski, and C. M. Marian, Chem. - Eur. J. 19, 17541 (2013).

${ }^{41}$ C. Sousa, C. de Graaf, A. Rudavskyi, and R. Broer, J. Phys. Chem. A 121, 9720 (2017).
${ }^{42}$ A. Rudavskyi, C. Sousa, C. de Graaf, R. W. A. Havenith, and R. Broer, J. Chem. Phys. 140, 184318 (2014).

${ }^{43}$ W. C. Hamilton, Acta Crystallogr. 15, 353 (1962).

${ }^{44}$ E. Roedern and T. R. Jensen, Inorg. Chem. 54, 10477 (2015).

${ }^{45}$ R. Eßmann, G. Kreiner, A. Niemann, D. Rechenbach, A. Schmieding, T. Sichla, U. Zachwieja, and H. Jacobs, Z. Anorg. Allg. Chem. 622, 1161 (1996).

${ }^{46}$ H. Jacobs, J. Bock, and C. Stüve, J. Less Common Met. 134, 207 (1987).

${ }^{47}$ L. C. Jackson, Philos. Mag. 4, 269 (1959).

${ }^{48}$ P. M. Champion and A. J. Sievers, J. Chem. Phys. 66, 1819 (1977).

${ }^{49}$ R. S. Rubins and H. R. Fetterman, J. Chem. Phys. 71, 5163 (1979).

${ }^{50}$ G. Agnetta, T. Garofano, M. B. Palma-Vittorelli, and M. U. Palma, Philos. Mag. 7, 495 (1962).

${ }^{51}$ C. Campochiaro, E. G. Pavel, and E. I. Solomon, Inorg. Chem. 34, 4669 (1995).

${ }^{52}$ D. C. Price, Can. J. Phys. 65, 1280 (1987).

${ }^{53}$ F. Neese, Wiley Interdiscip. Rev.: Comput. Mol. Sci. 2, 73 (2012).

${ }^{54}$ F. Neese, Wiley Interdiscip. Rev.: Comput. Mol. Sci. 8, el327 (2018).

${ }^{55} \mathrm{~L}$. McGhee, R. M. Siddique, and J. M. Winfield, J. Chem. Soc. Dalton Trans. 1988, 1309

${ }^{56}$ R. B. Wilson and E. I. Solomon, J. Am. Chem. Soc. 102, 4085 (1980).

${ }^{57}$ E. Buhks, G. Navon, M. Bixon, and J. Jortner, J. Am. Chem. Soc. 102, 2918 (1980).

${ }^{58}$ C. L. Xie and D. N. Hendrickson, J. Am. Chem. Soc. 109, 6981 (1987).

${ }^{59}$ J. K. McCusker, A. L. Rheingold, and D. N. Hendrickson, Inorg. Chem. 35, 2100 (1996).

${ }^{60}$ K. F. Purcell, J. Am. Chem. Soc. 101, 5147 (1979).

${ }^{61}$ E. König, Complex Chem (Springer Berlin Heidelberg, 1991), pp. 51-152.

${ }^{62} \mathrm{~J}$. K. McCusker, H. Toftlund, A. L. Rheingold, and D. N. Hendrickson, J. Am. Chem. Soc. 115, 1797 (1993).

${ }^{63}$ C. Musewald, P. Gilch, G. Hartwich, F. Pöllinger-Dammer, H. Scheer, and M. E. Michel-Beyerle, J. Am. Chem. Soc. 121, 8876 (1999).

${ }^{64}$ P. Gilch, C. Musewald, and M. E. Michel-Beyerle, Chem. Phys. Lett. 325, 39 (2000).

${ }^{65}$ Y. Ogawa, T. Ishikawa, S. Koshihara, K. Boukheddaden, and F. Varret, Phys. Rev. B 66, 073104 (2002).

${ }^{66}$ A. Hauser, Coord. Chem. Rev. 111, 275 (1991).

${ }^{67}$ A. Hauser, A. Vef, and P. Adler, J. Chem. Phys. 95, 8710 (1991).

${ }^{68}$ S. Schenker, A. Hauser, W. Wang, and I. Y. Chan, J. Chem. Phys. 109, 9870 (1998).

${ }^{69}$ W. Zhang, R. Alonso-Mori, U. Bergmann, C. Bressler, M. Chollet, A. Galler, W. Gawelda, R. G. Hadt, R. W. Hartsock, T. Kroll, K. S. Kjær, K. Kubiček, H. T. Lemke, H. W. Liang, D. A. Meyer, M. M. Nielsen, C. Purser, J. S. Robinson, E. I. Solomon, Z. Sun, D. Sokaras, T. B. van Driel, G. Vankó, T.-C. Weng, D. Zhu, and K. J. Gaffney, Nature 509, 345 (2014).

${ }^{70}$ K. Zhang, R. Ash, G. S. Girolami, and J. Vura-Weis, J. Am. Chem. Soc. 141, 17180 (2019).

${ }^{71}$ G. Auböck and M. Chergui, Nat. Chem. 7, 629 (2015).

${ }^{72}$ M. Bacci, Coord. Chem. Rev. 86, 245 (1988). 